\title{
Spectral evolution of the slowest classical nova V723 Cassiopeiae in the decline stage ${ }^{\star}$
}

\author{
T. Iijima
}

\author{
Astronomical Observatory of Padova, Asiago Section, Osservatorio Astrofisico, 36012 Asiago (Vi), Italy \\ e-mail: iijima@astras.pd.astro.it
}

Received 4 August 2005 / Accepted 15 November 2005

\section{ABSTRACT}

The spectral evolution of an extremely slow nova V723 Cas was monitored at Asiago Observatory for seven years. Observations in the long pre-maximum stage, which lasted more than four months, were reported in our previous paper. Here, we report the spectral evolution in the decline stage from January 1996 to December 2001. The spectra just after maximum luminosity showed prominent emission lines of H I, He I, Fe II, Ti II, Si II, Na I, etc. P Cygni type absorption components appeared on H I, He I, and Fe II lines about two months after maximum when the nova performed the second brightening. The radial velocities of the absorption components of $\mathrm{H} \mathrm{I}$ and He I lines varied from $-600 \mathrm{~km} \mathrm{~s}^{-1}$ in February to $-1600 \mathrm{~km} \mathrm{~s}^{-1}$ in June 1996. Broad emission wings of H I and He I lines that were red-shifted by about $1000 \mathrm{~km} \mathrm{~s}^{-1}$ were detected in the same season. When the third brightening began in July 1996, a new absorption system of H I and He I appeared in which the blue-shift changed from $-1000 \mathrm{~km} \mathrm{~s}^{-1}$ in July 1996 to $-1600 \mathrm{~km} \mathrm{~s}^{-1}$ in February 1997. Prominent emission lines of highly ionized ions such as [Fe VI], [Fe VII], and [Ca V] emerged in the period between May 30 and July 1, 1997. This nova probably entered the nebular stage in this period. The interval from maximum luminosity to the beginning of nebular stage is about 18 months, which is still longer than those of the other extremely slow novae: 10 months of RR Pic or 8 months of HR Del. Some emission lines of [Fe IV], which were not found on the spectra of usual classical novae, were detected on the spectra in the nebular stage. The ionization state grew with time to have a coronal emission line [Fe X] $\lambda 6375$ in November 1999 and later. The interstellar extinction is estimated at $E(B-V)=0.57 \pm 0.05$ and the distance to the nova at about $d=2.8 \mathrm{kpc}$, while the mass of the ejecta may be about $5 \times 10^{-6} M_{\odot}$. The abundance of helium is estimated at $N(\mathrm{He}) / N(\mathrm{H})=0.18 \pm 0.01$. The abundance of oxygen is not so different from those of usual classical novae, while a rather low abundance of nitrogen is noticed. The abundance of neon is roughly comparable to that of a neon nova QU Vul.

Key words. stars: individual: V723 Cassiopeiae - novae, cataclysmic variables - ISM: general

\section{Introduction}

Nova (V723) Cas 1995 was discovered on August 24, 1995 (Yamamoto 1995) as a star with $m_{\text {Vis. }} \cong 9.2$. After the long pre-maximum stage, which lasted about four months, the nova reached maximum luminosity, $m_{V} \cong 7.1$, in the middle of December 1995 (e.g., Munari et al. 1996; Ohshima et al. 1996). A detailed report of the spectral evolution in the pre-maximum stage was made in our previous paper (Iijima et al. 1998: hereafter Paper I).

Iijima \& Rosino (1997) preliminally reported that the nova entered the nebular stage in the period between May 30 and July 1, 1997, because numerous emission lines of highly ionized ions such as [Fe VI], [Fe VII], and [Ca V] emerged between the two dates. Zhu \& Hang (1999) also detected some emission lines of [Fe VII] and [Ca V] on an optical spectrum taken in December 1997. Kamath \& Ashok (1999) carried out infrared photometry and found that probably there was no dust

* Tables 1-3, 6-8 and Figs. 21, 22 are only available in electronic form at http://www. edpsciences.org condensation in the decline stage. Evans et al. (2003) made infrared observations in the period from 1996 to 1998 that supported the result of Kamath \& Ashok (1999). Rudy et al. (2002) made spectroscopic observations in the near-infrared region $(0.8-2.5 \mu \mathrm{m})$ in August 1999 and July 2000, and found some emission lines of highly ionized ions, such as [Al IX], [Ca VII], [Fe VI], and [Ti VII], but did not find any coronal line, e.g., [S IX] or [Fe XII]. Goranskij et al. (2000) suggested that this nova is probably a short-period ( 0.65325 day) binary system.

This paper presents the results of medium and high dispersion spectroscopy carried out at Asiago Observatory during the decline stage. Some properties of the nova are studied using the spectra in the nebular stage.

\section{Observations}

High dispersion spectra covering the range $\lambda \lambda 4330-6900 \AA$ or $\lambda \lambda 4600-7100 \AA$ with a resolution of $\lambda / \Delta \lambda \cong 8000$ were taken using a Reosc Echelle spectrograph mounted on the $182 \mathrm{~cm}$ telescope at the Mount Ekar station of the Astronomical 
Observatory of Padova. Medium dispersion spectra were taken using a Boller \& Chivens grating spectrograph or a prismatic spectrograph Camera VI. The Boller \& Chivens spectrograph was mounted on the $182 \mathrm{~cm}$ telescope until March 1998 then was translated onto the $122 \mathrm{~cm}$ telescope of the Astrophysical Observatory of the University of Padova at Asiago. The prismatic spectrograph had been mounted on the $122 \mathrm{~cm}$ telescope until the Boller \& Chivens was translated on it. The resolution of the Boller \& Chivens spectrograph is $\lambda / \Delta \lambda \cong 800$ with a grating of 600 lines $\mathrm{mm}^{-1}$. The dispersion of the prismatic spectrograph is about $300 \AA \mathrm{mm}^{-1}$ at $\mathrm{H} \alpha$ and $100 \AA \mathrm{mm}^{-1}$ at $\mathrm{H} \beta$. The full extent of the medium dispersion spectra covered with various grating or prism angles is $\lambda \lambda 3400-7180 \AA$, but usually only some parts of the range were observed each night. The reductions of the spectra were carried out using the standard tasks of the NOAO IRAF ${ }^{1}$ package at the Asiago Observatory. The spectrophotometric calibrations were made with the spectra of standard stars taken in the same nights, while HD17520 was used for the calibrations of the highdispersion spectra and Hiltner 102 for the medium-dispersion spectra. A log of the observations is given in Table 1, where UT is universal time at the start of exposure and "days" is number of days from maximum luminosity.

\section{Interstellar extinction and distance to the nova}

Some different values for the interstellar extinction to this nova have been reported in previous works. Gonzalez-Riestra et al. (1996) obtained $E(B-V)=0.6$ from the IUE observation of the $2200 \AA$ absorption band. Ohshima et al. (1996) found the same amount for the extinction by $U B V$ photometry in the pre-maximum stage. Chochol \& Pribulla (1997) estimated $E(B-V)=0.55$ from $U B V$ colors soon after maximum, but they used $E(B-V)=0.57$ in their work by taking an average of their result and that of Gonzalez-Riestra et al. (1996). Munari et al. (1996) estimated $E(B-V)=0.45$ from the equivalent widths of the interstellar absorption components of $\mathrm{Na} I \mathrm{D} 1$ and D2 lines. In Paper I, we derived $A_{V}=0.89$, namely $E(B-V)=0.29$, from an analysis of the galactic distribution of interstellar medium in the field of the nova. Rudy et al. (2002) have recently obtained $E(B-V)=0.2$ and 0.25 from the intensity ratios of hydrogen Paschen or Branckett lines in the infrared region.

In the present work, we found a mean intensity ratio of $\mathrm{H} \alpha$ to $\mathrm{H} \beta, I(\mathrm{H} \alpha) / I(\mathrm{H} \beta)=5.0 \pm 0.1$, on the spectra taken in and later than 1998 (see Sect. 6.1). Comparing this ratio with the theoretical one given by Hummer \& Storey (1987), $I(\mathrm{H} \alpha) / I(\mathrm{H} \beta)=2.70$ at $T_{\mathrm{e}}=11000 \mathrm{~K}$ and $N_{\mathrm{e}}=10^{8} \mathrm{~cm}^{-3}$ (see Sect. 7.1), we found $E(B-V)=0.57 \pm 0.05$. This result lies between those of Gonzalez-Riestra et al. (1996) and Ohshima et al. (1996) and the first result of Chochol \& Pribulla (1997). The difference from our previous result (Paper I) may be due to additional circumstellar obscure matter ejected by the nova itself. If we assume that the circumstellar medium is different from the standard interstellar medium in the gas-to-dust ratio

\footnotetext{
${ }^{1}$ IRAF is distributed by NOAO for Research in Astronomy, Inc. under cooperative agreement with the National Science Foundation.
}

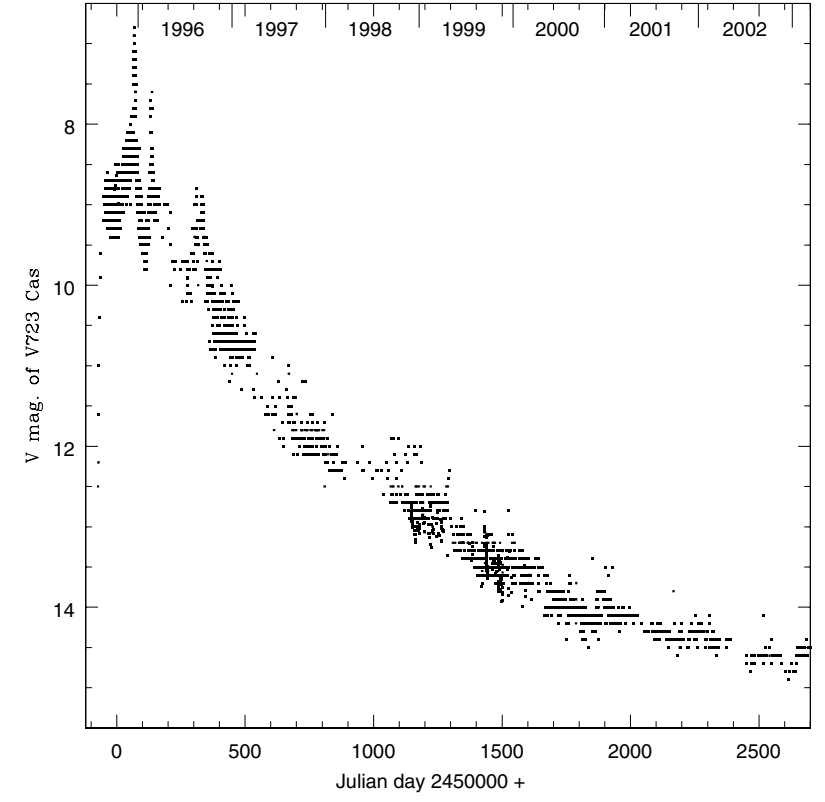

Fig. 1. Light curve of V723 Cas collected in VS-NET (http:// www.kusastro.kyoto-u.ac.jp/vsnet/index.html).

and/or in the abundance of sodium, it will be possible to explain the deviation of the result of Munari et al. (1996). On the contrary, it seems to be difficult to explain the disagreement between our result and those of Rudy et al. (2002), because theirs are also derived using the intensity ratios of $\mathrm{HI}$ lines in the nebular stage. The disagreement between our new result and those of Rudy et al. (2002) is an open question at the present time. In any case, we adopt $E(B-V)=0.57$ in this work.

The light curve of this nova collected in VS-NET is shown in Fig. 1. The time and magnitude of maximum luminosity were estimated at $V_{\max }=7.1$ on December 17,1995 (JD 2450 069.3). It is difficult to estimate the absolute magnitude of maximum luminosity from the light curve, because there were two secondary brightenings in 1996 (Fig. 1). This nova faded by about $2.5 \mathrm{mag}$ for 40 days after maximum. The number of days for a two-magnitude fading at that time was about 17. This fast decline rate, however, appeared to be inconsistent with the extremely slow evolution of this nova in the later stage. We made a strong smoothing of the light curve except for the points around maximum, and estimated the time for a three-magnitude fading $t_{3} \sim 230$ days, then converted it to the time for a two-magnitude fading, $t_{2} \sim 153$ days, using the formula of Warner (1995). The absolute magnitude of maximum luminosity is estimated at $M_{V}(\max ) \cong-6.9$ using the formula of Della Valle \& Livio (1995). Since the observed magnitude of maximum luminosity was $V=7.1$, we have a distance to the nova of about $2.8 \mathrm{kpc}$. Because of the ambiguity in the decline rate, it is difficult to estimate the error in the distance.

Chochol \& Pribulla (1997) measured the time for a threemagnitude fading and found $t_{3} \sim 173$ days. We do not adopt this result, because the nova largely brightened again after that time (Fig. 1). Evans et al. (2003) removed the three peaks from the light curve and obtained $m_{\mathrm{Vis}}(\max )=8.25$ and $t_{3} \sim 778$ days. We do not adopt these results either, because such a modification of light curve was not made in previous 


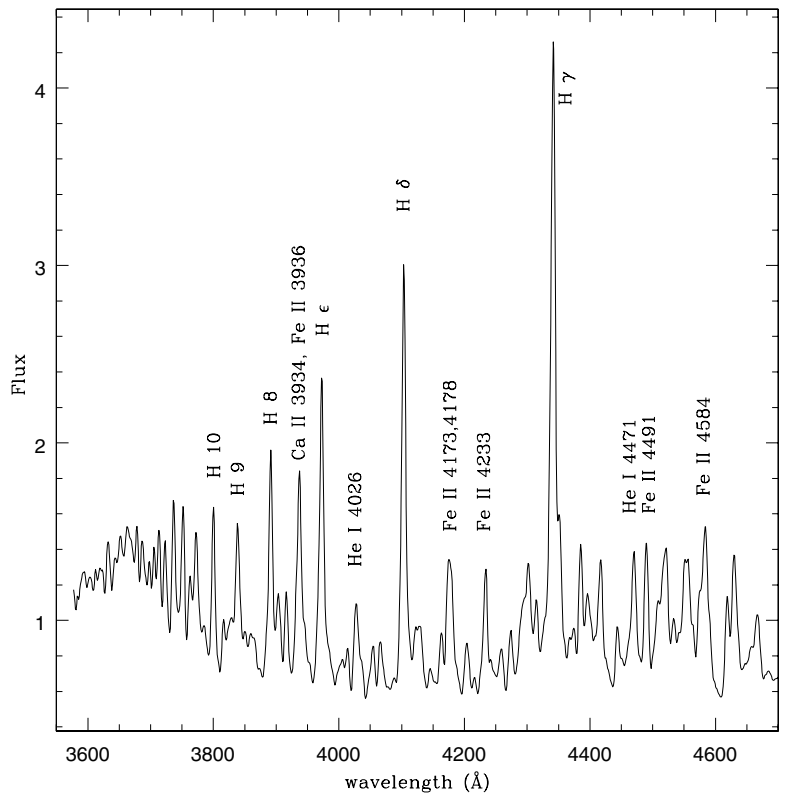

Fig. 2. A grating spectrum of V723 Cas on Jan. 2, 1996. The unit of ordinate is $10^{-12} \mathrm{erg} \mathrm{cm}^{-2} \mathrm{~s}^{-1} \AA^{-1}$.

works for the relation between the decline rate and the absolute magnitude of novae. Our distance is somewhat larger than that of Chochol \& Pribulla (1997), $2.39 \mathrm{kpc}$, while it is smaller than the $4.0 \mathrm{kpc}$ of Evans et al. (2003). Hachisu \& Kato (2004) have obtained a distance modulus $(m-M)_{V}=13.9$, when comparing their theoretical model with the observed light curve. If the reddening is $E(B-V)=0.57$ and $R=3.1$, the distance is $2.7 \mathrm{kpc}$. Their result agrees well with our estimate.

The precise distance will be able to measure from direct images of the expanding envelope. The expanding velocity of the ejecta is estimated at about $305 \mathrm{~km} \mathrm{~s}^{-1}$ from the widths of $\mathrm{H} \beta$ and He II 4686 emission lines in the nebular stage (see Sect. 6.5). Therefore, the diameter of the nebulosity is about 0.4 arcsecond at the present time (2005), if the distance is roughly $2.8 \mathrm{kpc}$. Thus, it will be possible to take direct images of the nebulosity by using the HST or a large telescope with adaptive optics.

\section{Spectral evolution}

\subsection{January-June 1996}

Figures 2-4 show tracings of our first spectra in the decline stage taken with the Boller \& Chivens spectrograph on January 2, 1996, that is, two weeks later and about two magnitudes below maximum.

Table 2 gives wavelengths in the heliocentric frame of the emission lines, their intensities relative to $\mathrm{H} \beta=100$, and identifications. The intensities after the correction of the interstellar extinction are presented. The interstellar extinction is corrected by using the task DEREDDEN of IRAF, namely, the extinction curve of Cardelli et al. (1989). We adopt $E(B-V)=0.57$ and $A_{V} / E(B-V)=3.1$. The same correction applies to all spectra studied in this article. The absolute intensity of $\mathrm{H} \beta$ is also given. The identifications are made using the multiplet table of

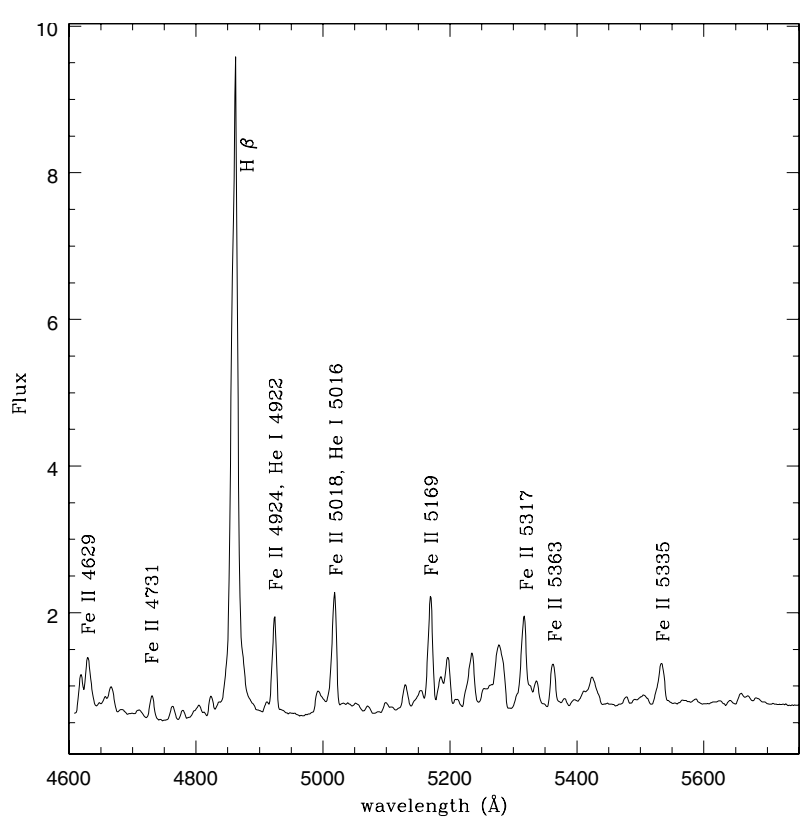

Fig. 3. A grating spectrum of V723 Cas on Jan. 2, 1996. The unit of ordinate is $10^{-12} \mathrm{erg} \mathrm{cm}^{-2} \mathrm{~s}^{-1} \AA^{-1}$.

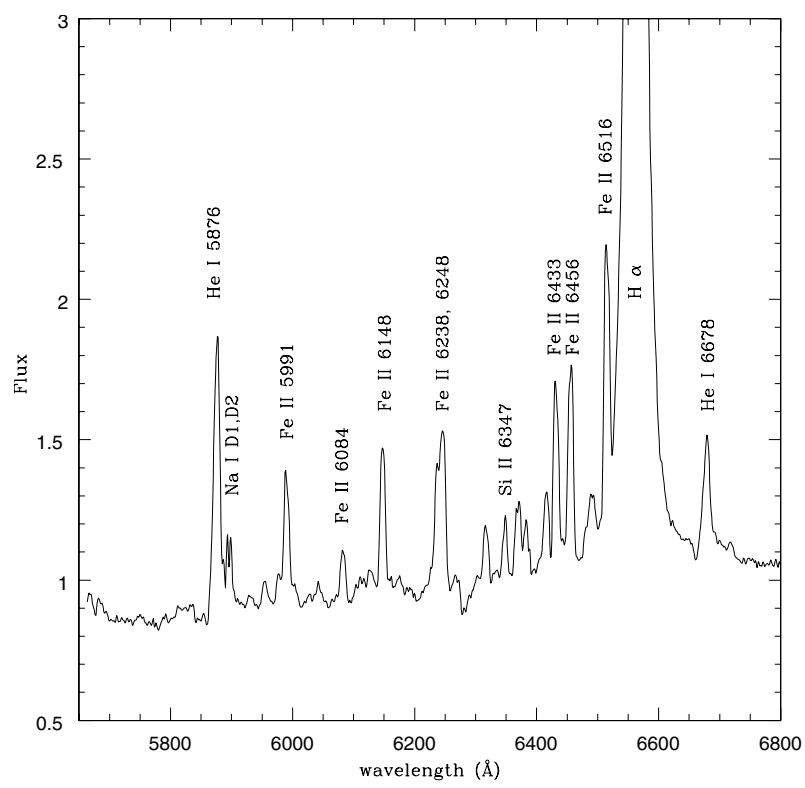

Fig. 4. A grating spectrum of V723 Cas on Jan. 2, 1996. The unit of ordinate is $10^{-12} \mathrm{erg} \mathrm{cm}^{-2} \mathrm{~s}^{-1} \AA^{-1}$.

Moore (1959), the catalog of emission lines of Meinel et al. (1968), and the list in the NIST atomic spectra database ${ }^{2}$. The term "unid" with wavelength in the column of identification means a line presented in the catalog of Meinel et al. (1968) without identification. The observational errors in the intensities are about $10 \%$, and the values of lower accuracy, which are mainly caused by blending with other lines, are denoted by a colon.

As seen in the figures, the prominent emission lines on the spectra are due to H I, He I, and Fe II. Numerous emission lines of singly ionized metals, Ti II, Cr II, Si II, Sc II, etc. are

${ }^{2}$ http://physics.nist.gov/cgi-bin/AtData/lines-form 


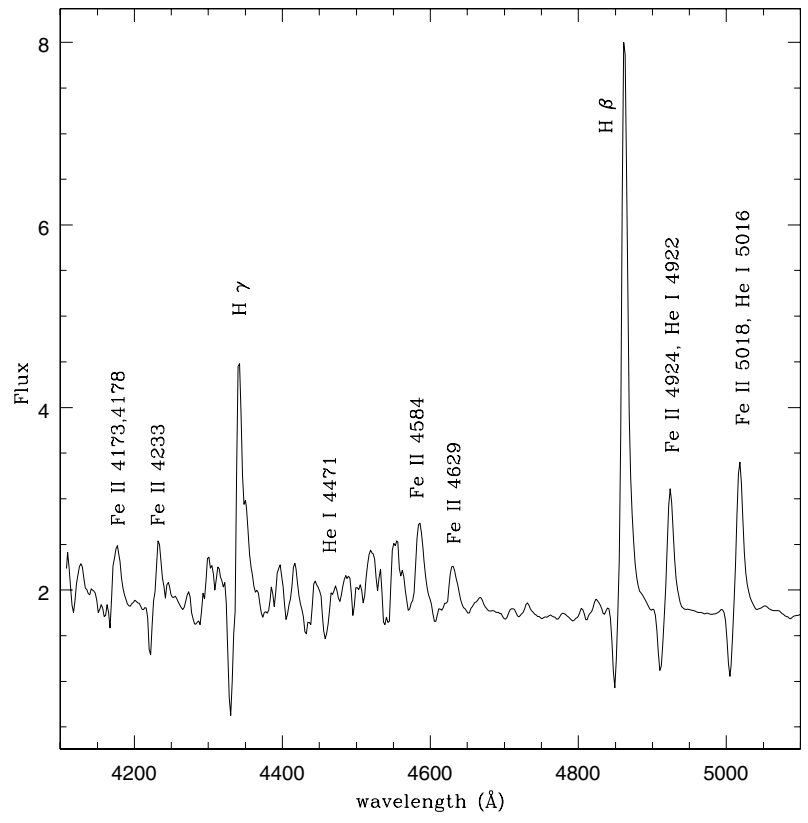

Fig. 5. A prismatic spectrum of V723 Cas on Feb. 17, 1996. The unit of ordinate is $10^{-12} \mathrm{erg} \mathrm{cm}^{-2} \mathrm{~s}^{-1} \AA^{-1}$.

also seen, and some emission lines of N II, [Fe II], [Ni II], and Na I D1 and D2 are detected (Table 2). The absence of the emission lines of [O I] and [N II] is noteworthy, because these lines usually appear prior to the emergence of He I emission lines in the early decline stage of classical novae (e.g., McLaughlin 1960).

When the second brightening started in February 1996 (Fig. 1), P Cygni type absorption components appeared on H I, $\mathrm{He}$ I, and Fe II lines. The absorption components developed around the peak of the second brightening on February 17, 1996 (Fig. 1). Figure 5 shows a tracing of a spectrum taken at the peak of the second brightening. The radial velocities of the absorption components are reported in Sect. 5.

Figure 6 shows a tracing of a red spectrum on March 2, 1996. The emission lines of N II $\lambda 5679.6$, [N II] $\lambda 5754.8$, and probably N II $\lambda 6242.5$ are newly noticed, while no emission line of [O I] is seen yet.

\subsection{July 1996-May 1997}

Figure 7 shows a tracing of our first high-dispersion spectrum in the decline stage taken on July 27, 1996 when the nova faded by about three magnitudes in the $V$ band. The prominent emission lines are due to H I, He I, Fe II, [Fe II], N II, and [N II]. Weak emission lines of [O I] $\lambda \lambda 6300$ and 6364 are detected, while no emission line of He II is seen. Since the sky was slightly cloudy in the night, intensities of the emission lines on this spectrum were not measured.

Figure 8 shows profiles of $\mathrm{H} \alpha, \mathrm{H} \beta, \mathrm{He} \mathrm{I} \lambda 5876$, and $\mathrm{Fe}$ II $\lambda 5316.6$ of the same spectrum where the ordinate is an arbitrary logarithmic scale. Each of $\mathrm{H} \mathrm{I}$ lines has a main absorption component blue-shifted by about $-1100 \mathrm{~km} \mathrm{~s}^{-1}$ and a secondary component blue-shifted by about $-770 \mathrm{~km} \mathrm{~s}^{-1}$ (see Sect. 5). The shape of the absorption component of He I $\lambda 5867$

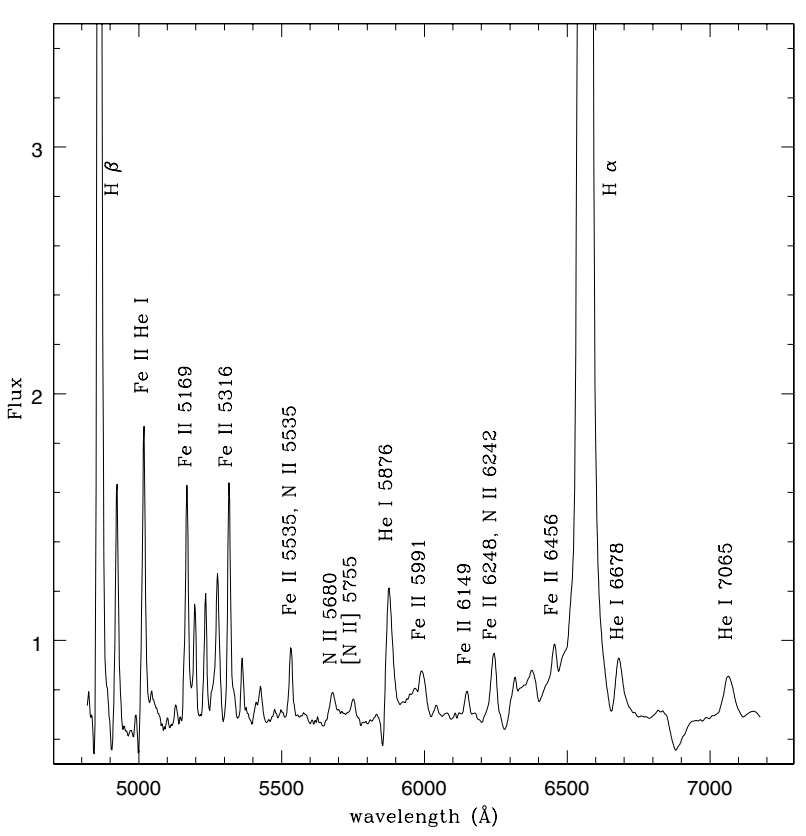

Fig. 6. A prismatic spectrum of V723 Cas on March 2, 1996. The unit of ordinate is $10^{-12} \mathrm{erg} \mathrm{cm}^{-2} \mathrm{~s}^{-1} \AA^{-1}$.

is different from those of $\mathrm{H} \mathrm{I}$ lines. Both hydrogen and helium lines have emission wings red-shifted by about $1000 \mathrm{~km} \mathrm{~s}^{-1}$.

The profile of Fe II $\lambda 5316.6$ represents the Fe II lines on the spectrum. The emission lines of Fe II are narrower than for $\mathrm{H} \mathrm{I}$ or $\mathrm{He} \mathrm{I}$, and no absorption component accompanies it. The weak emission feature in the red side of Fe II $\lambda 5316.6$ is not its red-shifted component, but is due to [Fe II] $\lambda 5333.7$. The profiles of Fe II lines are different from those of $\mathrm{H}$ I or $\mathrm{He}$ I lines on the same spectrum, but are nearly identical with most emission lines in the nebular stage (see Sect. 6.5).

The profiles of $\mathrm{H} \mathrm{I}$ and $\mathrm{He}$ I lines suggest that the emitting regions of these lines were not spherically symmetric, but at least a part of them had the form of jets. On the other hand, the emission lines of Fe II may have been emitted in a more extended region, where the expanding velocity was low. The structure of the ejecta seems to have been very complicated at this stage, and a three-dimensional analysis is awaited.

Figure 9 shows a tracing of a spectrum taken on January 28, 1997, when the nova faded by about four magnitudes. Wavelengths, intensities relative to $\mathrm{H} \beta=100$, and identifications of the emission lines are given in Table 3 . The notations of Table 3 are the same as for Table 2 . The prominent emission lines are of H I, He I, [N II], [Fe II], [O III], N II, N III, and Si II, while most of the emission lines of Fe II have disappeared. The emission line of He II $\lambda 4686$ is seen, but is still much weaker than $\mathrm{H} \beta$. A weak trace of the emission line of [O I] $\lambda 6300$ is seen on this spectrum.

Broad emission humps are seen at $\lambda \lambda 4620-4650 \AA$ and $\lambda \lambda 5650-5690 \AA$ (Fig. 9). The former may be due to N III $\lambda \lambda 4634.2,4640.6,4641.6$; [Fe II] $\lambda 4640.6$; and N II $\lambda \lambda 4613.9$ and 4630.5, while N II $\lambda \lambda 5666.6,5676.0$, 5679.6, and 5686.2 may be the main contributors to the second hump. It seems that the nitrogen flush (see e.g., McLaughlin 1960) occurred in this period, but the intensities of these humps 

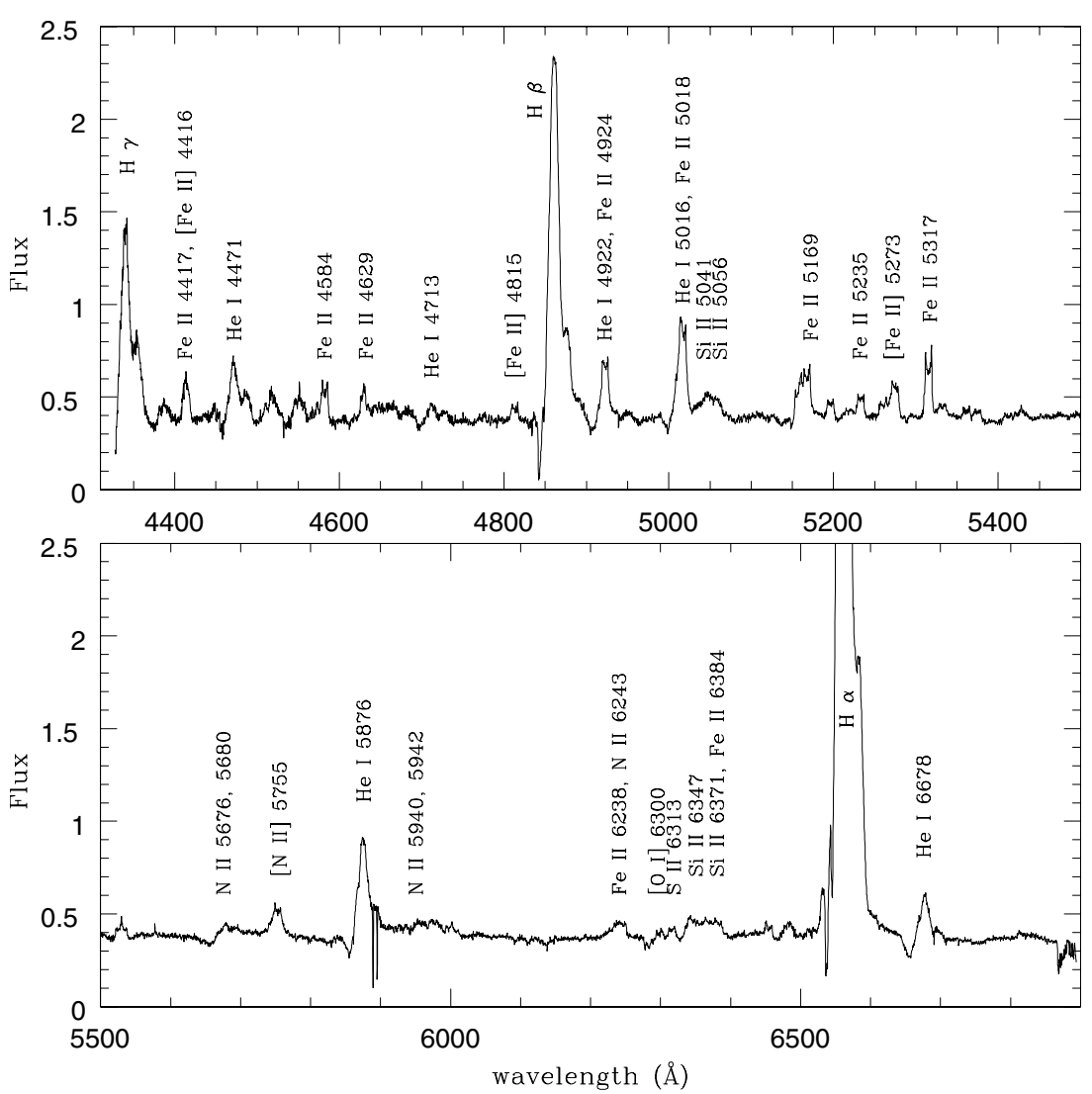

Fig. 7. An echelle spectrum of V723 Cas on July 27, 1996. The unit of ordinate is $10^{-12} \mathrm{erg} \mathrm{cm}^{-2} \mathrm{~s}^{-1} \AA^{-1}$.

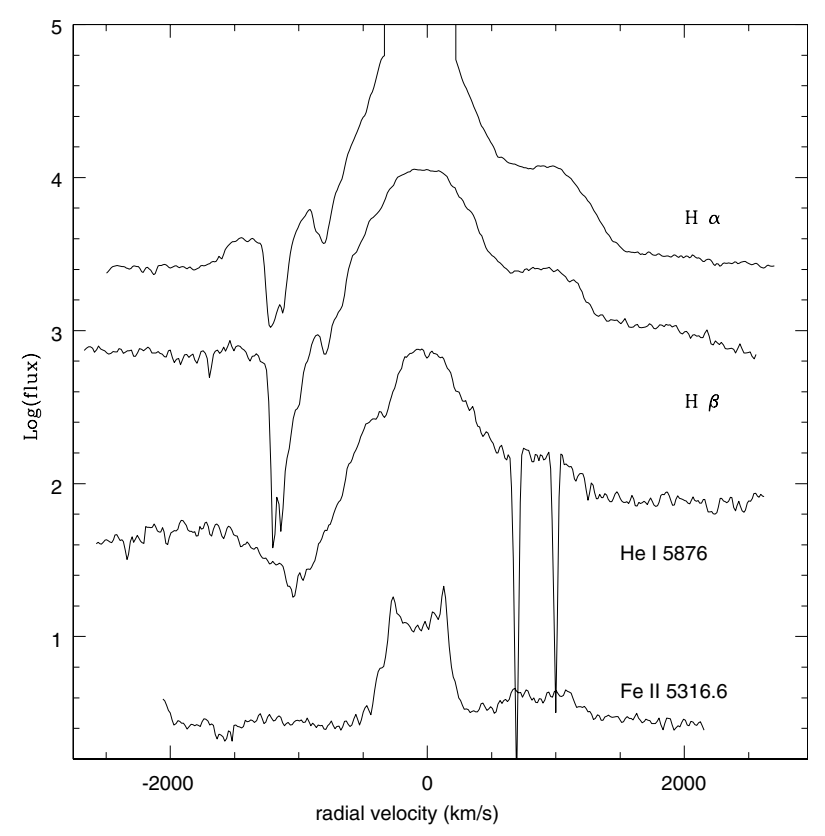

Fig. 8. Profiles of $\mathrm{H} \alpha, \mathrm{H} \beta$, He I $\lambda 5876$, and Fe II $\lambda 5316.6$ on July 27 , 1996. The ordinate is an arbitrary logarithmic scale.

were much lower than those of the usual classical novae at the same stage (see e.g., V1494 Aql: Iijima \& Esenoglu 2003: hereafter Paper II). The intensities of these broad emission humps relative to $\mathrm{H} \beta$ remained nearly stable during the period from December 1996 to February 1997, then they had significantly faded in May 1997.
It is hard to see any absorption components of $\mathrm{H}$ I or of the singlet He I line at $\lambda 6678$ on the spectrum, while the triplet He I lines at $\lambda \lambda 5876$ and 7065 show clear absorption components (Fig. 9). The red-shifted emission wings of $\mathrm{H} \mathrm{I}$ and He I lines are still visible.

\subsection{June 1997-2001}

Large changes of the spectral feature occurred in the period between May 30 and July 1, 1997. The tracings of the spectra taken on these dates are shown in Figs. 10 and 11. The intensities of prominent emission lines relative to $\mathrm{H} \beta$ are given in Table 4. For the purpose of reducing the size of the table, first two figures of the wavelengths in the column of identification are omitted. As seen in the figures, the emission line of He II $\lambda 4686$ strengthen dramatically between the two dates. The emission lines of [O III] are seen already on the earlier spectrum (Fig. 10), but many emission lines of highly ionized ions such as [Fe VI], [Fe VII], [Ca V], etc. are first detected on the latter spectrum (Fig. 11, Table 4). The emission lines of [O I] have disappeared on May 30, 1997 (Fig. 10). The emissions of Na I D1 and D2 on the spectra are of a night sky. Since the emission lines due to the city lights were very strong on these spectra, we were not able to subtract them perfectly from the tracings of the stellar spectra.

The nebular emission line [O III] $\lambda 5007$ was much weaker than $\mathrm{H} \beta$ throughout the period of our monitoring, so we cannot use the intensity of the [O III] line as an indicator of the beginning of the nebular stage. Considering the large changes in the spectral features, we decided that the nova entered the 

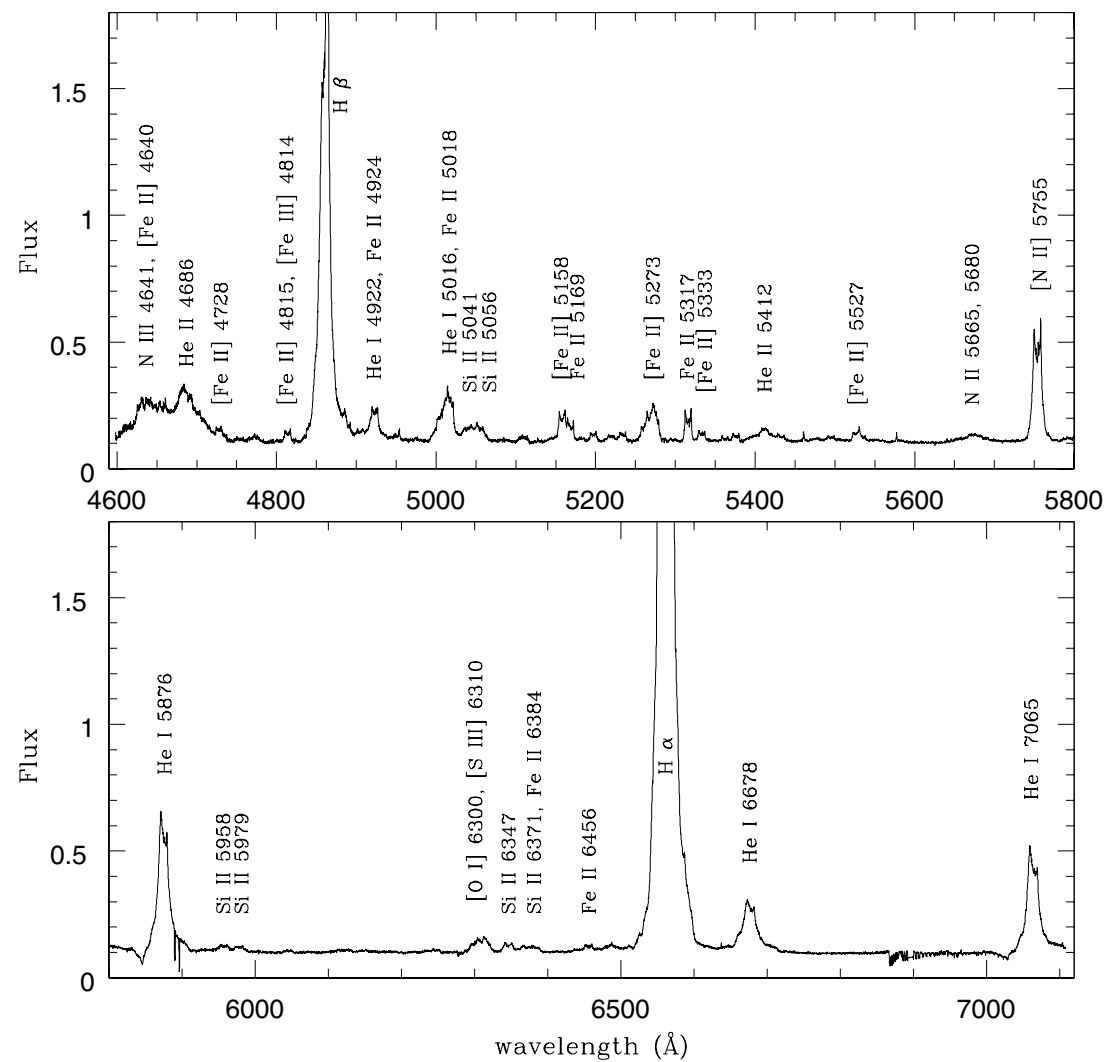

Fig. 9. An echelle spectrum of V723 Cas on Jan. 28, 1997. The unit of ordinate is $10^{-12} \mathrm{erg} \mathrm{cm}^{-2} \mathrm{~s}^{-1} \AA^{-1}$.

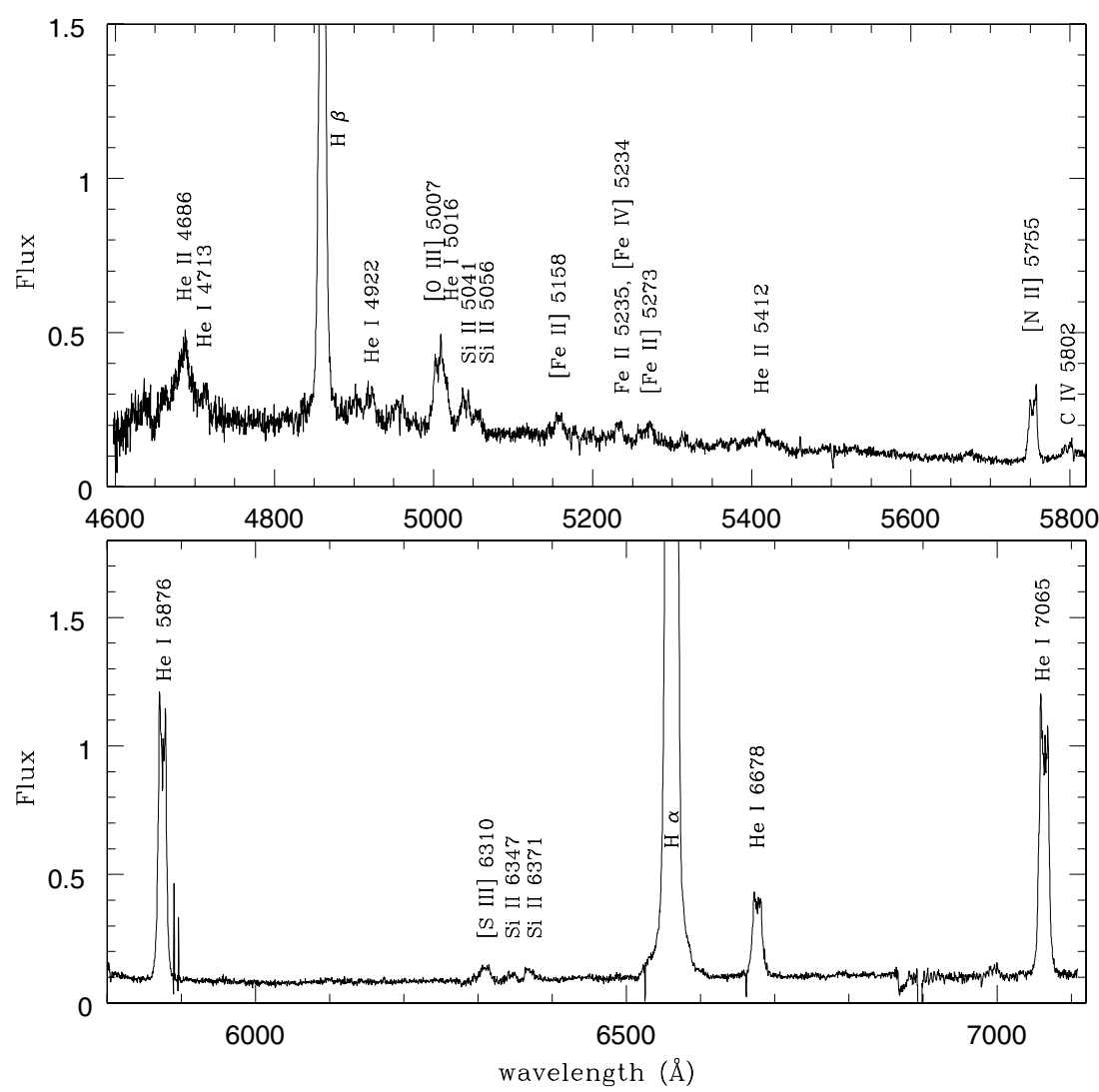

Fig. 10. An echelle spectrum of V723 Cas on May 30, 1997. The unit of ordinate is $10^{-12} \mathrm{erg} \mathrm{cm}^{-2} \mathrm{~s}^{-1} \AA^{-1}$.

nebular stage between May 30 and July 1, 1997. The interval from maximum luminosity to the beginning of nebular stage is, therefore, about one year and six months. The well-observed, extremely slow nova HR Del entered its nebular stage in the 8th month from maximum (Rafanelli \& Rosino 1978). The other extremely slow nova, RR Pic, showed a sudden strengthening of He II lines in March 1926, that is about 10 months 


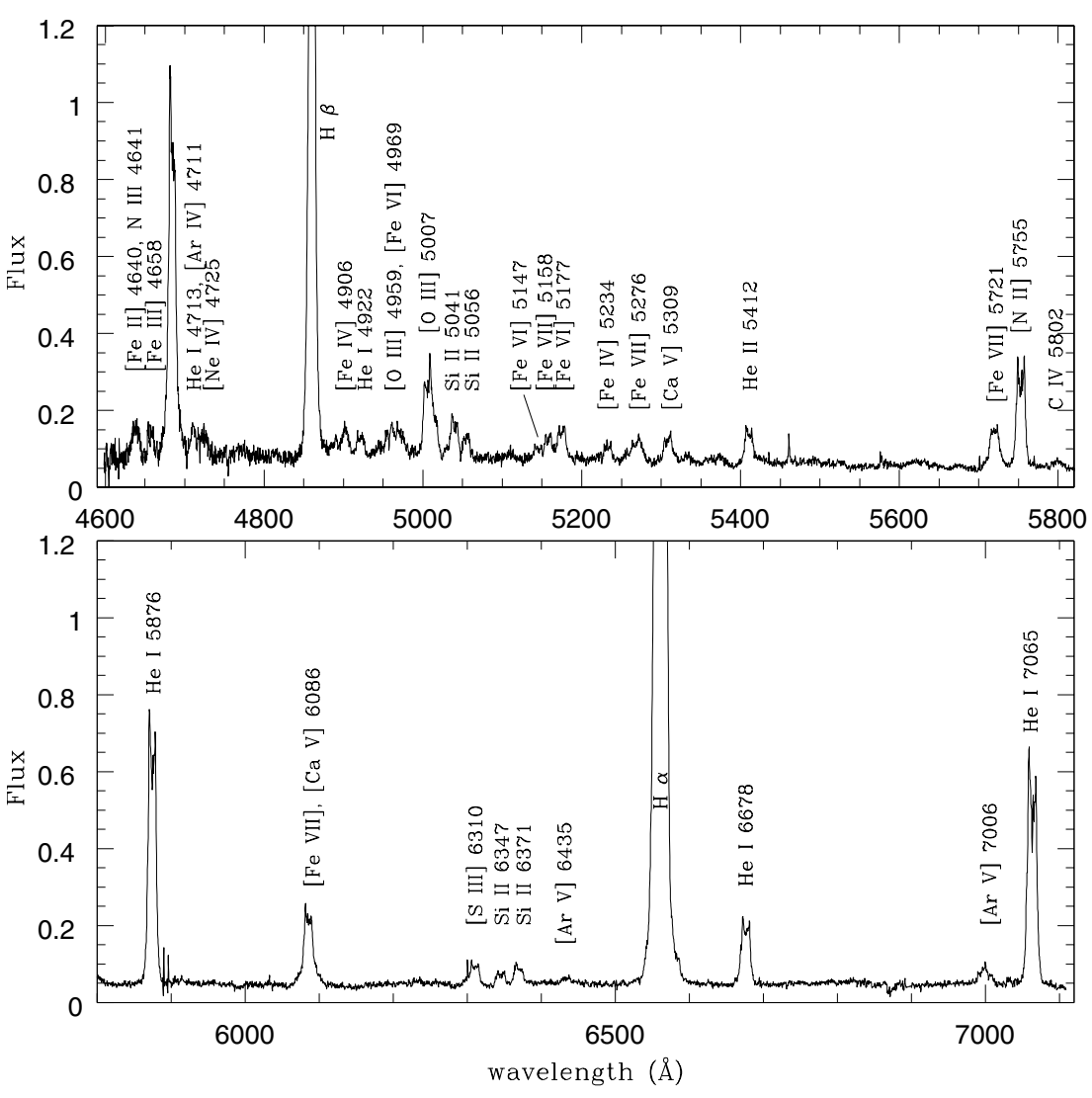

Fig. 11. An echelle spectrum of V723 Cas on July 1, 1997. The unit of ordinate is $10^{-12} \mathrm{erg} \mathrm{cm}^{-2} \mathrm{~s}^{-1} \AA^{-1}$. after maximum (Jones 1931). Thus, V723 Cas is the nova of the slowest spectral evolution in the history.

Evans et al. (2003) report that the emission line [Al V] $\lambda 2.904 \mu \mathrm{m}$ was first detected on July 3, 1997 and that those of the other highly ionized ions such as [S IX], [Si VI], [Al IX], and $[\mathrm{Ca} \mathrm{VII}]$ in the infrared region were detected in November 1997. Their results are consistent with ours. On the other hand, Evans et al. (2003) suggested that they might have detected [S IX] $\lambda 1.258 \mu \mathrm{m}$ on June 11,1996 and [Ca IV] $\lambda 3.206 \mu \mathrm{m}$ on December 18, 1996. In our observations, however, no emission line of highly ionized ion was detected until July 1, 1997.

The ionization state grew with time throughout the nebular stage to have a coronal emission line [Fe X] $\lambda 6375$ in November 1999 and later. Detailed reports of the spectra in the nebular stage are given in Sect. 6.

\section{Absorption components of $\mathrm{H} \mathrm{I}$, He I, and Fe II lines}

The radial velocities of the absorption components of $\mathrm{H} \mathrm{I}$, Fe II, Si II, etc. in the pre-maximum stage are reported in Paper I. The mean radial velocity of the absorption components of Fe II lines changed from $-270 \mathrm{~km} \mathrm{~s}^{-1}$ in August 1995 to $-160 \mathrm{~km} \mathrm{~s}^{-1}$ in December 1995 at several days before maximum (Paper I).

Our first spectra in the decline stage showed no clear absorption feature (Figs. 2-4). The P Cygni type absorption features of H I, He I, and Fe II were first detected on February 7, 1996 at the beginning of the second brightening (Sect. 4.1). The heliocentric radial velocities of the absorption components are given in Table 5 and shown in Fig. 12. The observational errors in the results obtained with the spectrograph Boller \& Chivens or the Camera VI are about $\pm 50 \mathrm{~km} \mathrm{~s}^{-1}$, and those obtained by the Echelle spectrograph are about $\pm 5 \mathrm{~km} \mathrm{~s}^{-1}$. The radial velocity of the nova system is estimated at $-58.4 \pm 0.9 \mathrm{~km} \mathrm{~s}^{-1}$ measuring the line center of some forbidden lines in the nebular stage (Sect. 6.5). Thus, it is necessary to subtract this velocity from the values of Table 5 to have the outflow velocity in the frame of the nova system. Since the lines of Fe II $\lambda \lambda 4923.9$ and 5018.4 $\AA$ are blended with He I $\lambda \lambda 4921.9$ and $5015.7 \AA$, respectively, only the line at $\lambda 4233.2 \AA$ is used for Fe II.

The radial velocity of Fe II line was somewhat different from those of $\mathrm{H} \mathrm{I}$ and He I lines; namely, the radial velocities of the absorption components of $\mathrm{H}$ I and He I lines changed by about $300 \mathrm{~km} \mathrm{~s}^{-1}$ during 20 days in February-March 1996, while that of the Fe II line changed by about $130 \mathrm{~km} \mathrm{~s}^{-1}$ (Table 5, Fig. 12). It is suggested in Sect. 4.2 that the emitting region of $\mathrm{Fe}$ II lines is different from $\mathrm{H} \mathrm{I}$ and $\mathrm{He} \mathrm{I}$ lines. The radial velocity variations suggest that also the absorption component of Fe II line is formed in a different region from those of $\mathrm{H} \mathrm{I}$ and $\mathrm{He} \mathrm{I}$ absorption lines.

As seen in Fig. 12, there is a jump in the radial velocities between June 6 and July 27, 1996 (JD 2450 240-291). A new system of absorption components of H I and He I seems to have appeared in July 1996: JD 2450280 (Fig. 12). Probably, a new mass ejection occurred on the third brightening whose peak was found in August 1996 (Fig. 1). The absorption components in and later than July 1996 may have depended on the new ejecta. 
Table 4. Intensities of emission lines relative to $\mathrm{H} \beta=100$ on May 30 and July $1,1997$.

\begin{tabular}{|c|c|c|c|}
\hline \multirow{2}{*}{$\begin{array}{c}\lambda_{\odot} \\
(\AA)\end{array}$} & \multirow[t]{2}{*}{ Identification } & \multicolumn{2}{|c|}{$\overline{I I(\lambda)}$} \\
\hline & & May 30 & July 1 \\
\hline 4638.7 & {$[\mathrm{Fe}$ II $]$ 2F, 39.7, N III 2, 40.6} & & 5.4 \\
\hline 4656.6 & {$[\mathrm{Fe}$ III $] 3 \mathrm{~F}, 58.1$} & 3.9 & 3.6 \\
\hline 4684.5 & He II, 85.7 & 16.7 & 56.9 \\
\hline 4712.8 & He I, 13.2, [Ar IV] 1F, 11.4 & $5.4^{*}$ & 3.7 \\
\hline 4724.6 & {$[\mathrm{Ne}$ IV] $1 \mathrm{~F}, 24.2,25.7$} & & 3.1 \\
\hline 4814.5 & {$[\mathrm{Fe}$ II $] 2 \mathrm{~F}, 14.6$} & & 1.2 \\
\hline 4860.7 & $\mathrm{H} \beta$ & 100 & 100 \\
\hline 4902.5 & {$[\mathrm{Fe}$ IV $]-, 06.2,[\mathrm{Fe} \mathrm{II}] 20 \mathrm{~F}, 05.4$} & & 3.0 \\
\hline 4921.3 & He I, 21.9 & 5.2 & 2.4 \\
\hline 4958.8 & [O III] $1 \mathrm{~F}, 58.9$ & & 3.0 \\
\hline 4970.0 & {$[\mathrm{Fe} \mathrm{VI}] 2 \mathrm{~F}, 68.8,74.0$} & & 3.5 \\
\hline 5005.8 & [O III] $1 \mathrm{~F}, 06.9$ & 13.3 & 10.6 \\
\hline 5014.8 & He I, 15.7 & 5.2 & 4.7 \\
\hline 5033.3 & {$[\mathrm{Fe}$ II $]$ 4F, 35.3, [Fe III] 2F, 32.7} & & 1.5 \\
\hline 5040.4 & Si II $5,41.1$ & 6.7 & 4.7 \\
\hline 5055.2 & Si II $5,56.0$ & 3.5 & 3.0 \\
\hline 5144.5 & {$[\mathrm{Fe} \mathrm{VI}] 2 \mathrm{~F}, 46.8$} & & 1.7 \\
\hline 5157.9 & {$[\mathrm{Fe}$ II] $18 \mathrm{~F}, 58.0$} & 2.8 & \\
\hline 5158.6 & {$[\mathrm{Fe}$ VII $] 2 \mathrm{~F}, 58.3$} & & 3.0 \\
\hline 5174.9 & {$[\mathrm{Fe} \mathrm{VI}] 2 \mathrm{~F}, 77.0$} & & 4.6 \\
\hline 5233.0 & {$[\mathrm{Fe}$ IV],- 33.8} & & 1.9 \\
\hline 5233.3 & $\mathrm{Fe}$ II $49,34.6$ & 1.9 & \\
\hline 5260.3 & {$[\mathrm{Fe}$ II] $19 \mathrm{~F}, 61.6$} & 1.6 & 0.6 : \\
\hline 5272.2 & {$[\mathrm{Fe}$ II] $18 \mathrm{~F}, 73.4$} & 2.5 & \\
\hline 5274.9 & {$[\mathrm{Fe} \mathrm{VII}] 2 \mathrm{~F}, 76.1$} & & 3.0: \\
\hline 5309.5 & {$[\mathrm{Ca} \mathrm{V}]$ 1F, 08.9} & & 3.2 \\
\hline 5314.7 & $\mathrm{Fe}$ II $49,16.6$ & 1.1 & \\
\hline 5376.8 & {$[\mathrm{Fe}$ II] $19 \mathrm{~F}, 76.5$} & 0.6 & \\
\hline 5410.7 & He II, 11.5 & 1.2 & 4.6 \\
\hline 5672.9 & Fe II -, 73.2 & 0.6 & \\
\hline 5720.2 & {$[\mathrm{Fe}$ VII $]$ 1F, 20.9} & & 5.4 \\
\hline 5753.4 & {$[\mathrm{~N}$ II] $3 \mathrm{~F}, 54.8$} & 7.8 & 12.0 \\
\hline 5800.0 & C IV $1,01.5$ & & 0.9 \\
\hline 5874.2 & He I, 75.6 & 40.9 & 28.9 \\
\hline 6085.6 & {$[\mathrm{Fe} \mathrm{VII}] 1 \mathrm{~F}, 85.5,[\mathrm{Ca} \mathrm{V}] 1 \mathrm{~F}, 85.9$} & & 10.7 \\
\hline 6310.0 & [S III] $3 \mathrm{~F}, 10.2$ & 2.7 & 2.4 \\
\hline 6345.3 & Si II $2,47.1$ & 1.1 & 1.4 \\
\hline 6369.5 & Si II $2,71.4$ & 1.9 & 2.1 \\
\hline 6433.8 & [Ar V] 1F, 34.9 & & 0.6 \\
\hline 6562.1 & $\mathrm{H} \alpha$ & 440 & 385 \\
\hline 6582.3 & {$[\mathrm{~N}$ II $] 1 \mathrm{~F}, 83.6$} & & 3.1 \\
\hline 6676.4 & He I, 78.1 & 12.6 & 6.9 \\
\hline 6999.1 & [Ar V] 1F, 06.3 & & 1.8 \\
\hline 7063.3 & He I, 65.2 & 34.4 & 20.7 \\
\hline
\end{tabular}

*: No [Ar IV] on May 30. Interstellar extinction is corrected by $E(B-V)=0.57$.

\section{Spectra in the nebular stage}

\subsection{Intensities of emission lines}

As mentioned in Sect. 4.3, the spectral features changed dramatically between May 30 and July 1, 1997, so we decided that the nova entered nebular stage in this period. The intensity ratio of $\mathrm{H} \alpha / \mathrm{H} \beta$, however, was much higher than the theoretical one (Hummer \& Storey 1987) even on the latter spectrum (Table 4). Similar high-intensity ratios of $\mathrm{H} \alpha / \mathrm{H} \beta$ were also observed on other novae, e.g., V1500 Cyg (Ferland 1978) and V1494 Aql (Paper II). Netzer (1975) showed that the selfabsorptions of Lyman and Balmer photons in optically thick nebulae presented such high-intensity ratios of $\mathrm{H}$ I lines. The same effect would explain the ratios of $\mathrm{H} \alpha / \mathrm{H} \beta$ of this nova in and earlier than 1997 . The ejected nebulosity probably became optically thin in 1998 , because the mean intensity ratio of $\mathrm{H} \alpha / \mathrm{H} \beta$ in and later than 1998 was $5.0 \pm 0.1$. This ratio is close to the one expected from the recombination theory (Hummer \& Storey 1987) when the interstellar extinction $E(B-V)=0.57$ is corrected. We, therefore, use the spectra taken in and later than 1998 for analysis of the ejecta properties (Sect. 7). 
Table 5. Radial velocities in $\mathrm{km} \mathrm{s}^{-1}$ of absorption components of H I, He I, Fe II, and N II lines.

\begin{tabular}{|c|c|c|c|c|c|c|c|c|c|c|c|c|c|}
\hline \multicolumn{2}{|l|}{ Date } & $\overline{\mathrm{JD}^{1}}$ & Inst. & $\mathrm{H} \delta$ & $\begin{array}{l}\text { Fe II } \\
4233\end{array}$ & $\mathrm{H} \gamma$ & $\begin{array}{l}\text { He I } \\
4471\end{array}$ & $\mathrm{H} \beta$ & $\begin{array}{r}\mathrm{N} \text { II } \\
5676\end{array}$ & $\begin{array}{r}\mathrm{He} \text { I } \\
5876\end{array}$ & $\mathrm{H} \alpha$ & $\begin{array}{r}\text { He I } \\
6078\end{array}$ & $\begin{array}{r}\mathrm{He} \mathrm{I} \\
7065\end{array}$ \\
\hline \multicolumn{14}{|l|}{1996} \\
\hline Feb. & 7 & 121.3 & C-VI & & & -640 & -710 & -740 & & & & & \\
\hline Feb. & 8 & 122.3 & " & & & -650 & $-670:$ & -690 & & & & & \\
\hline Feb. & 14 & 128.3 & " & & -850 & -770 & -780 & -800 & & & & & \\
\hline Feb. & 17 & 131.3 & " & & -870 & -770 & -900 & -790 & & & & & \\
\hline Feb. & 28 & 142.3 & " & & -1000 & -970 & -1050 & -1080 & & & & & \\
\hline March & 1 & 144.3 & " & & -950 & -980 & -1040 & -1080 & & & & & \\
\hline March & 5 & 148.3 & " & & -980 & -1030 & -1070 & -1130 & & & & & \\
\hline March & 14 & 157.3 & " & & & -1050 & -1070 & -1120 & & & & & \\
\hline June & 6 & 240.6 & $B \& C$ & & & -1450 & -1370 & -1530 & & & & & \\
\hline July & 27 & 291.6 & Ech & & & & -913 & -1117 & -1100 & -1016 & -1163 & -1041 & \\
\hline July & 27 & 291.6 & " & & & & & $-777 \dagger$ & & & $-802 \dagger$ & & \\
\hline $\begin{array}{l}\text { Dec. } \\
1997\end{array}$ & 15 & 433.4 & $\mathrm{C}-\mathrm{VI}$ & & & & & & & -1480 & & & -1580 \\
\hline Jan. & 18 & 467.3 & C-VI & & & & -1750 & & & & & & \\
\hline Jan. & 28 & 477.3 & Ech & & & & & & & -1605 & & & \\
\hline Jan. & 29 & 478.4 & " & & & & & & & -1645 & & & \\
\hline Feb. & 10 & 490.3 & $B \& C$ & -1680 & & & -1790 & & & -1690 & & & \\
\hline Feb. & 20 & 500.3 & Ech & & & & & & & -1740 & & & \\
\hline
\end{tabular}

$\mathrm{JD}^{1}$ : Julian date $-2450000 ; \uparrow$ : secondary absorption component.

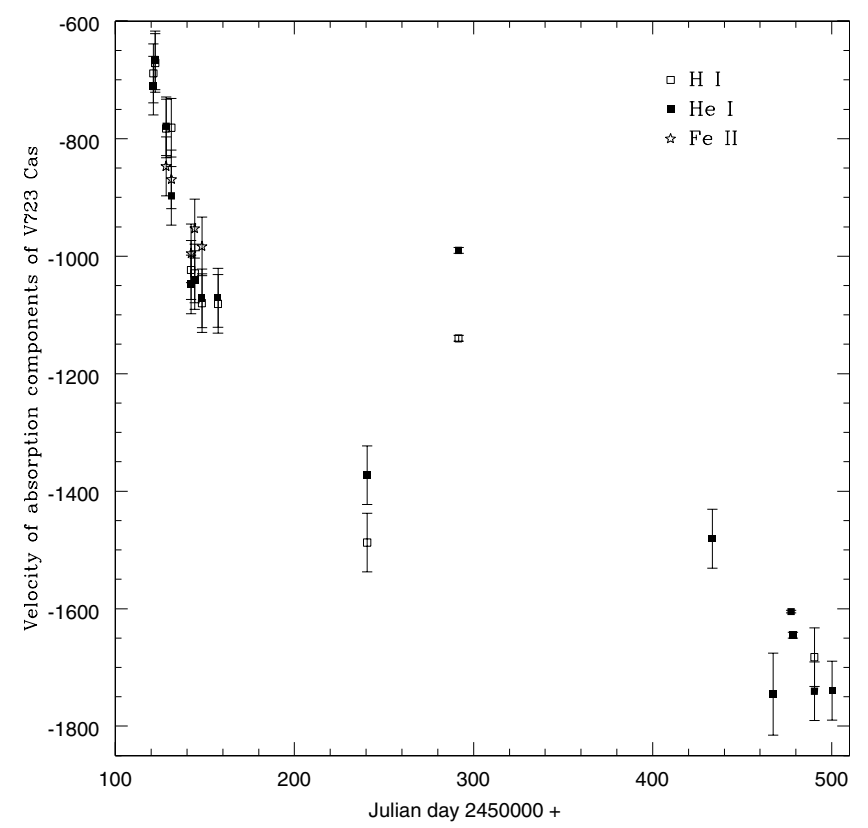

Fig. 12. Radial velocities of the absorption components of the $\mathrm{H} \mathrm{I}$, $\mathrm{He}$ I, and Fe II lines of V723 Cas. The unit of ordinate is $\mathrm{km} \mathrm{s}^{-1}$.

Table 6 gives identifications for the emission lines and their intensities relative to $\mathrm{H} \beta=100$ measured on the highdispersion spectra taken in and later than 1998. Table 7 gives the results obtained with the medium-dispersion spectra in 1998 and 1999, while those in 2000 and 2001 are presented in Table 8. Some results in Tables 7 and 8 have two observation dates. Those are averages of two or more spectra taken on subsequent days. This was done, because the spectral variation was expected to have been sufficiently slow during this stage. Some spectra presented in Table 8 do not extend throughout the spectral region of $\mathrm{H} \beta$. Intensities of the emission lines in such spectra are given relative to $\mathrm{H} \alpha=270$. To reduce the size of the tables, the multiplet numbers and first two figures of the wavelengths in the column of identification are omitted. The absolute intensities of the $\mathrm{H} \beta$ emission line are measured on the medium-dispersion spectra. A tracing of a high-dispersion spectrum taken on August 10, 1998 is shown in Fig. 13, and the medium-dispersion spectra of blue, green, and red regions taken on October 16, 1998 are shown in Figs. 14-16. Figure 17 shows a high-dispersion spectrum taken on September 29, 1999, and Figs. 18-20 show the mediumdispersion spectra taken on November 28, 29, and 30, 1999. The bluest part of the spectrum on November 28, 1999 is shown separately in Fig. 21 (Sect. 6.3).

\section{2. [Fe IV] lines}

It is interesting that the emission line of [Fe IV] at $\lambda 5233.8 \AA$ and possibly also the one at $\lambda 4906.2 \AA$ are detected on the spectra of this nova in the nebular stage (Figs. 11, 13, 17, 19; Tables $4,6,7)$. The emission line at about $\lambda 5233 \AA$ on the spectra in the earlier stage is identified with Fe II 49, $\lambda 5234.6$ (Tables 2-4). On the other hand, it is unlikely that the emission line at $25233.0 \AA$ (Tables 4, 6) or at $25232.3 \AA$ (Table 7) in the nebular stage is due to the same element, because no other emission line of Fe II is detected on the same spectra. Therefore, the identification with [Fe IV] $\lambda 5233.8 \AA$ is more reasonable for that line in the nebular stage. It seems that there is another emission line of [Fe IV] at $\lambda 4906.2 \AA$ 


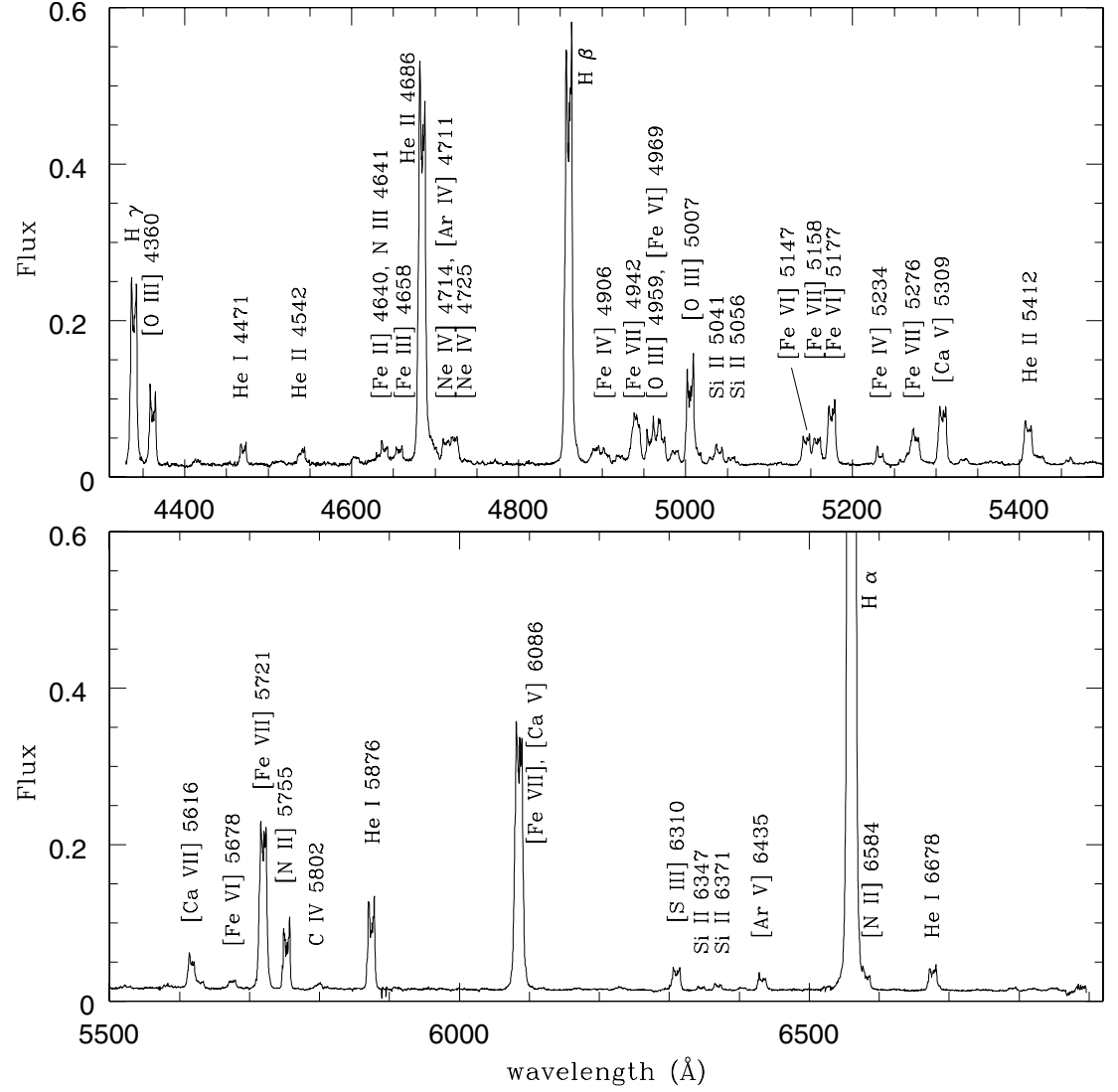

Fig. 13. An echelle spectrum of V723 Cas on August 10, 1998. The unit of ordinate is $10^{-12} \operatorname{erg~cm}^{-2} \mathrm{~s}^{-1} \AA^{-1}$.

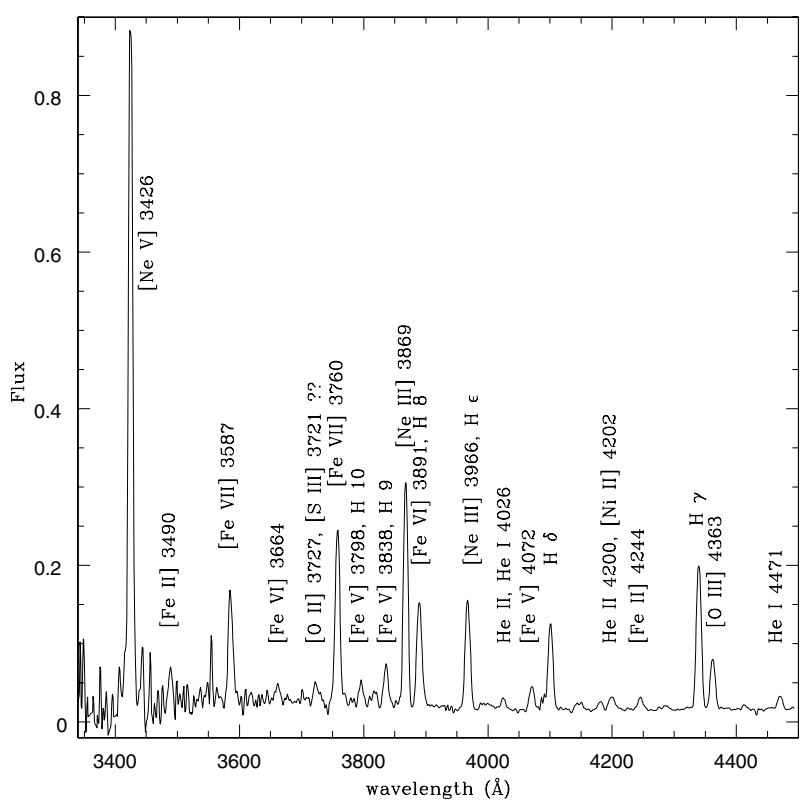

Fig. 14. A grating blue spectrum of V723 Cas on Oct. 16, 1998. The unit of ordinate is $10^{-12} \mathrm{erg} \mathrm{cm}^{-2} \mathrm{~s}^{-1} \AA^{-1}$.

(Tables 4, 6, 7). This identification, however, is not established in this work, because of the blending with [Fe II] $\lambda 4905.4$.

The emission lines of [Fe IV] have not been observed on the spectra of the usual classical novae, but were seen on the spectra of a symbiotic nova RR Tel (Thackeray 1977). Probably the extremely slow nova RR Pic also showed the emission line of [Fe IV] 15233.8 , because Jones (1931) found a weak

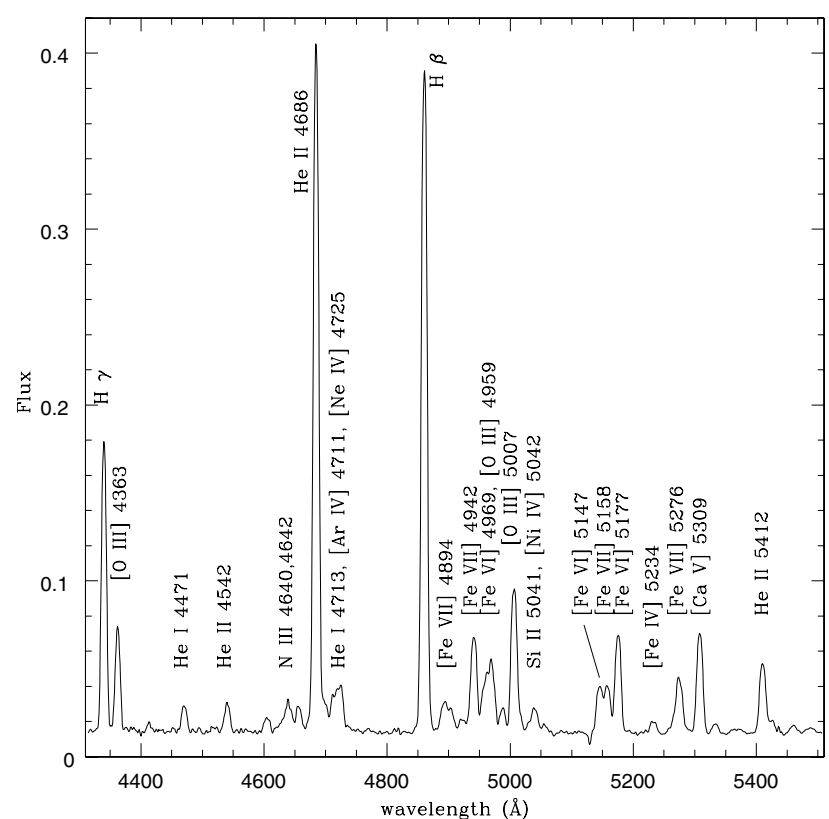

Fig. 15. A grating green spectrum of V723 Cas on Oct. 16, 1998. The unit of ordinate is $10^{-12} \mathrm{erg} \mathrm{cm}^{-2} \mathrm{~s}^{-1} \AA^{-1}$.

emission line at $\lambda 5232.5 \AA$ on the spectra in 1926. Jones (1931) himself suggested an identification with Fe II 49, $\lambda 5234.6$ for that line, because forbidden lines of [Fe IV] were not known in his epoch. His identification, however, is doubtful, because the stronger lines of Fe II in the same multiplet at $\lambda \lambda 5276.0$ and $5316.6 \AA$ (Moore 1959) were not seen on the same 


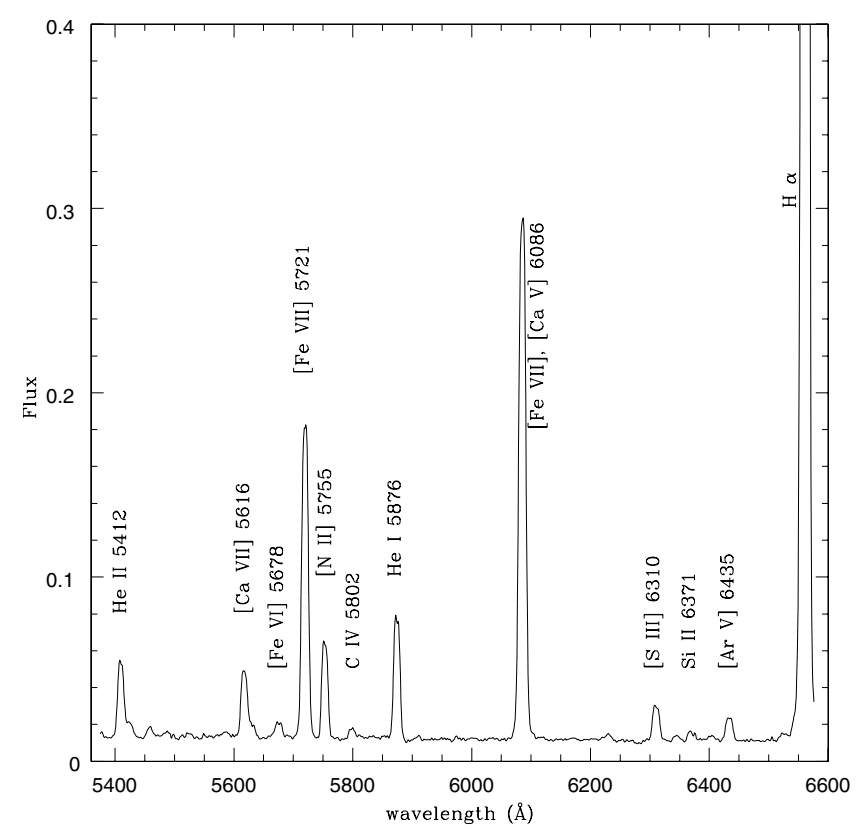

Fig. 16. A grating red spectrum of V723 Cas on Oct. 16, 1998. The unit of ordinate is $10^{-12} \mathrm{erg} \mathrm{cm}^{-2} \mathrm{~s}^{-1} \AA^{-1}$.

spectra. The emission line at $\lambda 5310.8 \AA$, which was identified with Fe II 49, $\lambda 5316.6$ by Jones (1931), should have been due to [Ca V] $\lambda 5308.9$, because the emission lines of highly ionized ions such as [Fe VII] and [Ca V] were prominent on the same spectra. It may be better to identify the emission line at $\lambda 5232.5 \AA$ of RR Pic with [Fe IV] $\lambda 5233.8$.

Rudy et al. (2002) found numerous emission lines on the infrared spectra of V723 Cas in the nebular stage whose wavelengths coincided with those of [Fe III], [Fe IV], or [Fe V]. They, however, were not able to establish their identifications, because of the absence of spectral data in the optical region. Our results do support their identifications.

\subsection{Emission line at $\lambda 3727 \AA$}

Figure 21 shows the bluest part of the medium-dispersion spectrum on November 28, 1999, where an emission line is seen at $\lambda 3727 \AA$ A This line has not been observed in the usual classical novae, at least as far as we know. For example, the ten classical novae studied by Williams et al. (1991) show no emission line of a nearby wavelength. On the other hand, emission lines with nearly the same wavelengths were observed in some symbiotic novae, one at $\lambda 3725.9 \AA$ of RR Tel (Thackeray 1977) and another at $\lambda 3727 \AA$ of V1016 Cyg and HM Sge (Schmid \& Schild 1990). Thackeray (1977) identified the line with O IV $\lambda 3725.8$ blended by He II $\lambda 3726$ and [O II] $\lambda 3726$, while Schmid \& Schild (1990) identified them with a blend of [O II] $\lambda \lambda 3726$, 3729 , and [S III] $\lambda 3721$.

The contribution of O IV $\lambda 3725.8$ is probably small in the present case, because the other line of O IV in the same multiplet at $\lambda 3726.8 \AA$ (Moore 1959) is very faint or absent in our spectrum. The line of [S III] $\lambda 3721$ could not be a candidate for the identification, because the disagreement between its laboratory wavelength and the observed one is much larger than the observational error. It is also difficult to identify this emission line with the blend of [O II] lines, because its intensity is too high with respect to those of [O III] lines on the same spectrum (see Sect. 7.5). There should be at least one more line of a highly ionized ion in the emission line. One candidate for the line is [Ni V] 23726.7 . The material is, however, not yet sufficient for us to establish this identification.

\subsection{Coronal line $[\mathrm{Fe} X] \lambda 6375$}

No trace of the coronal emission line $[\mathrm{Fe} \mathrm{X}] \lambda 6375$ was seen until September 1999 (Fig. 17), but it was first detected at the end of November 1999 (Fig. 20), and its emission line was blended by Si II $\lambda 6371$ on the first spectra. The intensity of the other emission line of Si II in the same multiplet at $\lambda 6374.1$ (Moore 1959) suggests that Si II $\lambda 6371$ was dominant in the blend in November 1999, while [Fe X] $\lambda 6375$ became dominant in February 2000 (Table 8). (The emission line Si II $\lambda 6374.1$ was blended with [K V] $\lambda 6349.5$ on these spectra, but the latter should have been weaker than the Si II line.) The contribution of Si II $\lambda 6371$ may have been negligible in 2001, because the blend of Si II + [K V] $\lambda 6345$ was not detected (Table 8). A tracing of a red spectrum taken on October 15, 2001 is shown in Fig. 22, where the coronal line [Fe X] $\lambda 6375$ is the third strongest emission line in the spectral region after $\mathrm{H} \alpha$ and $[\mathrm{Fe} \mathrm{VII]}+[\mathrm{Ca} \mathrm{V}] \lambda 6086$.

We are not able to know whether there were other coronal lines, e.g., [Fe XIV] $\lambda 5303$ or [Fe XI] $\lambda 7892$, because our last spectra did not extend to the spectral ranges of these lines. However, the other coronal lines are expected to have been absent, because the infrared coronal line [Fe XIII] $\lambda \lambda 10747$ or 10798 was not detected in July 2000 (Rudy et al. 2002).

It is known that some recurrent novae show coronal emission lines on outbursts (see e.g., McLaughlin 1960). A systematic search for coronal lines in the spectra of classical novae was made by McLaughlin (1953), who found the emission line of [Fe X] $\lambda 6375$ on the spectra of CP Pup, V603 Aql, etc. Among recent novae, GQ Mus (1983) showed an unusually strong emission line of $[\mathrm{Fe} \mathrm{X}] \lambda 6375$, which was stronger than $\mathrm{H} \alpha$ in the final stage of the spectral evolution (Krautter \& Williams 1989; Pèquignot et al. 1993). Also V1500 Cyg (Ferland et al. 1977) and V1494 Aql (Paper II) showed an $[\mathrm{Fe} \mathrm{X}] \lambda 6375$ emission line.

All the above-mentioned novae are fast novae. At least, as we know, no slow nova showed the coronal line [Fe X] $\lambda 6375$. Some infrared coronal lines, e.g., [Al IX] $\lambda 2.04 \mu \mathrm{m}$ and [Si IX] $\lambda 3.92 \mu \mathrm{m}$, were detected on the spectra of a slow nova QU Vul (Greenhouse et al. 1988), but the optical coronal line [Fe X] $\lambda 6375$ was probably not detected or at most was very weak (Saizar et al. 1992). It is noteworthy that we have only three well-observed extremely slow novae at the present time, but two of them, HR Del (e.g., Rafanelli \& Rosino 1978) and V723 Cas (this paper), show the emission line of [Fe X] $\lambda 6375$. A detailed study of coronal lines of classical novae is beyond the scope of this paper, and it seems fairly difficult to explain why fast novae and extremely slow novae show the $[\mathrm{Fe} \mathrm{X}]$ line, but normally slow novae do not. 

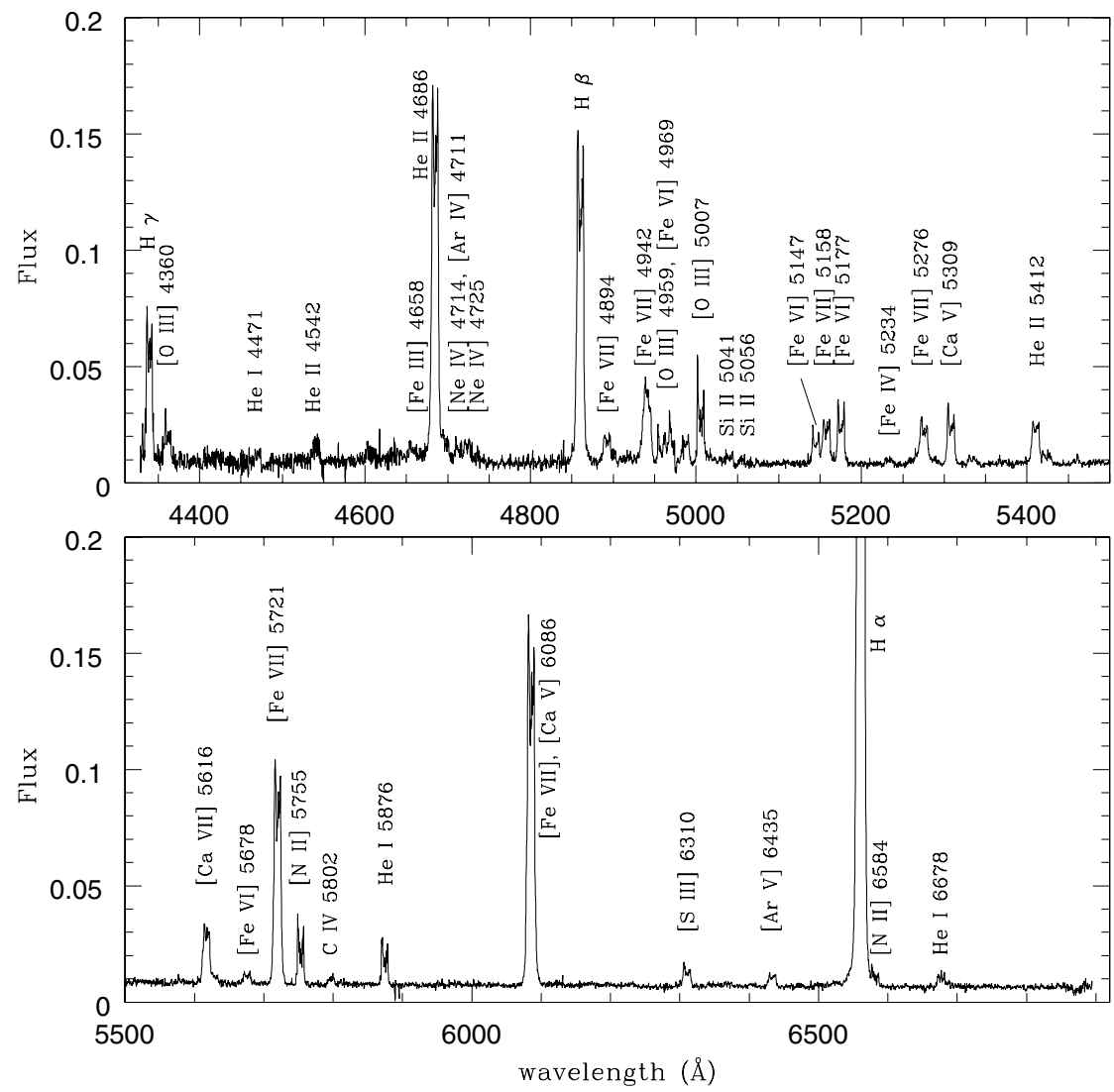

Fig. 17. An echelle spectrum of V723 Cas on Sept. 29, 1999. The unit of ordinate is $10^{-12} \mathrm{erg} \mathrm{cm}^{-2} \mathrm{~s}^{-1} \AA^{-1}$.

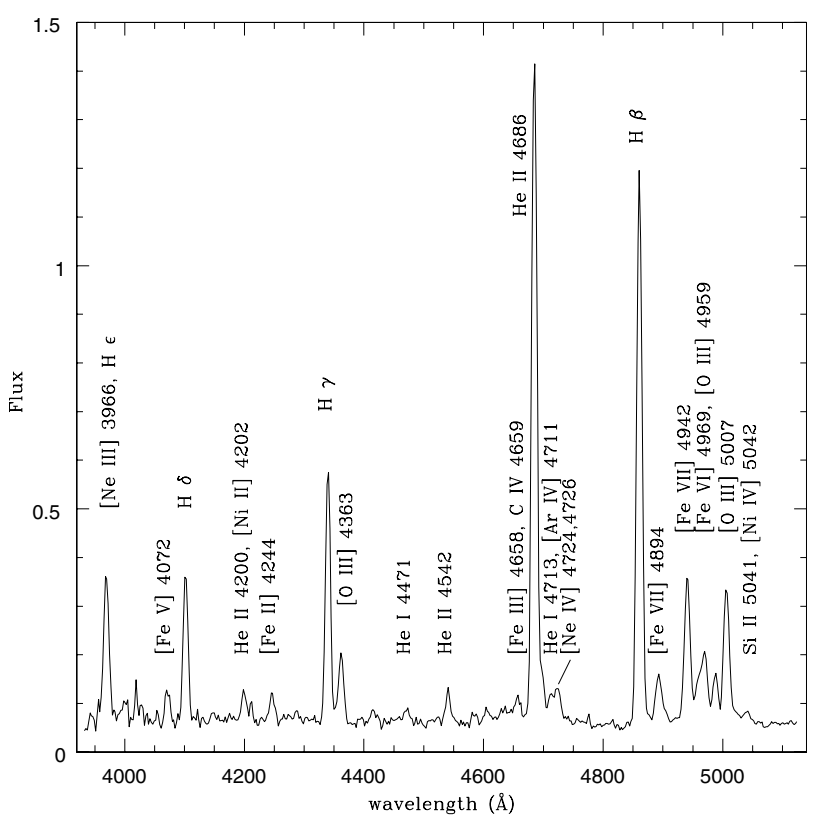

Fig. 18. A grating blue spectrum of V723 Cas on Nov. 28, 1999. The unit of ordinate is $10^{-13} \mathrm{erg} \mathrm{cm}^{-2} \mathrm{~s}^{-1} \AA^{-1}$.

\subsection{Profiles of prominent emission lines}

Figure 23 shows profiles of the emission lines of [Fe VII] 15721, [N II] $\lambda 5755$, and He I $\lambda 5876$ on December 27, 1998. Profiles of He II $\lambda 4686, \mathrm{H} \beta$, and [OIII] $\lambda 5007$ on the same spectrum are shown in Fig. 24. The ordinates of the figures are intensity scales, but zero levels of the tracings are arbitrarily

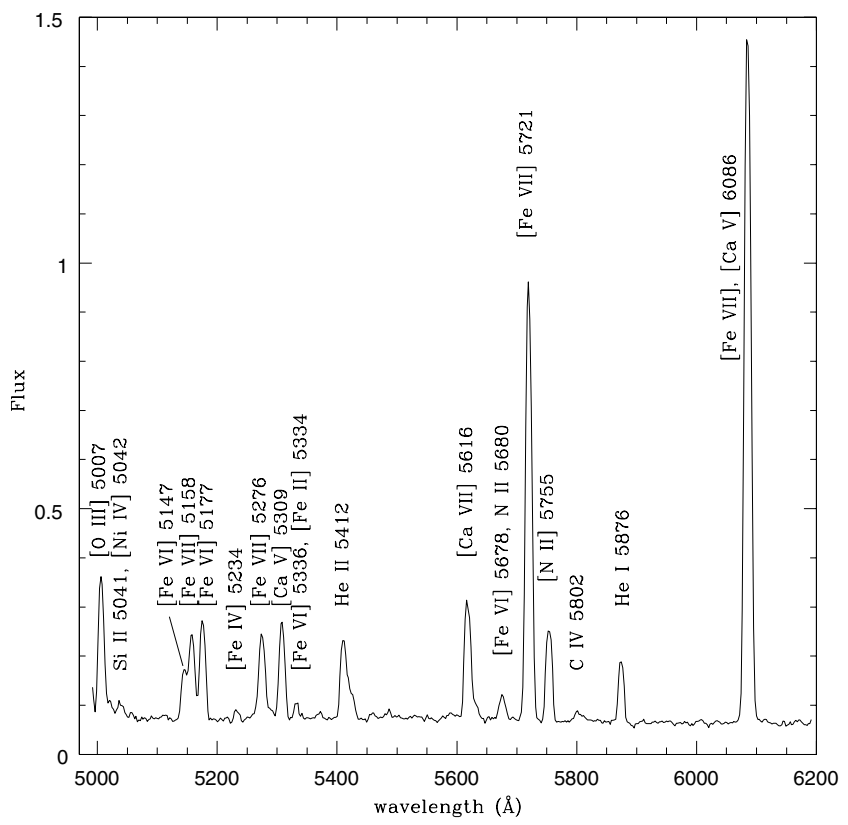

Fig. 19. A grating green spectrum of V723 Cas on Nov. 29, 1999. The unit of ordinate is $10^{-13} \mathrm{erg} \mathrm{cm}^{-2} \mathrm{~s}^{-1} \AA^{-1}$.

shifted. The tracing of [O III] in Fig. 24 is multiplied by a factor 1.5. Each of these emission lines consists of a main body and a blue-shifted weak wing. The main emission bodies show castellated profiles with six or seven peaks. The mean interval between the bluest and reddest peaks of the main bodies of these lines is $440 \pm 5 \mathrm{~km} \mathrm{~s}^{-1}$. The full widths at half maxima 


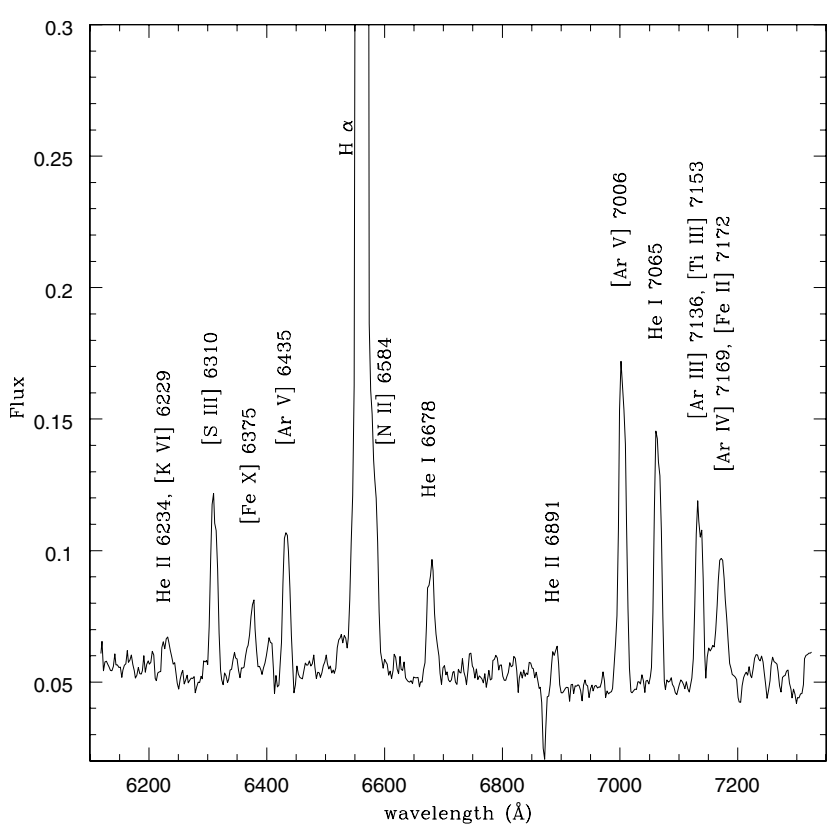

Fig. 20. A grating red spectrum of V723 Cas on Nov. 30, 1999. The unit of ordinate is $10^{-13} \mathrm{erg} \mathrm{cm}^{-2} \mathrm{~s}^{-1} \AA^{-1}$.

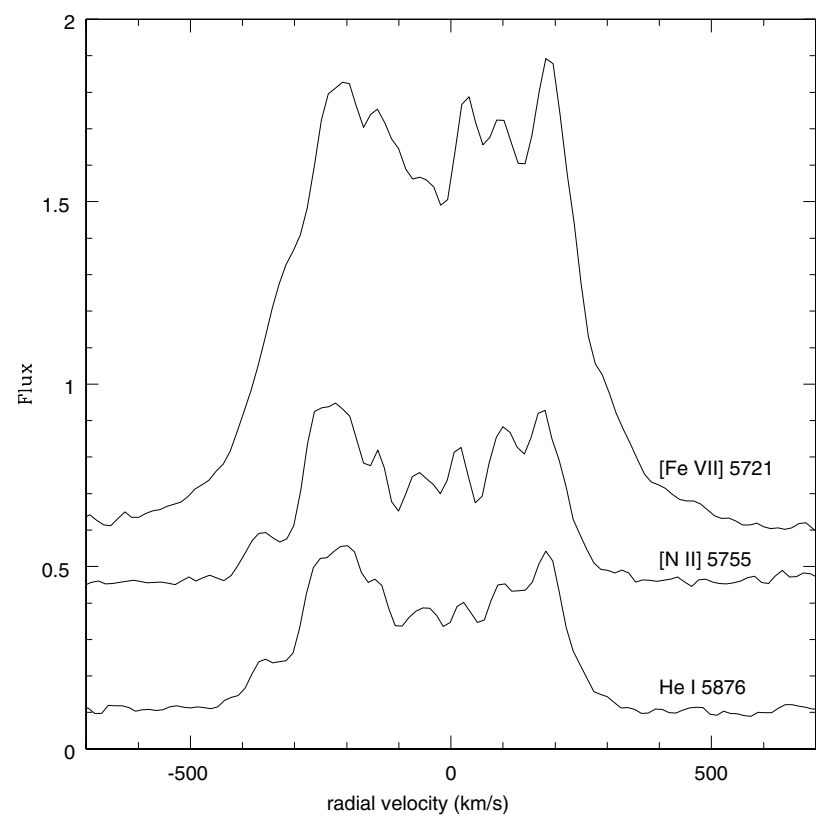

Fig. 23. Profiles of [Fe VII] 5721, [N II] 5755, and He I 5876 on December 27, 1998. The ordinate is an intensity scale.

of HeII $\lambda 4686$ and $\mathrm{H} \beta$ are $610 \pm 5 \mathrm{~km} \mathrm{~s}^{-1}$. The mean radial velocity of the center of the main emission bodies of the forbidden lines is $-58.4 \pm 0.9 \mathrm{~km} \mathrm{~s}^{-1}$, which may reflect the radial velocity of the nova system.

The profiles of the emission lines are somewhat different from one another. For example, the bluest peak is higher than the reddest one in [O III] and He II, while the reddest peak is higher in [Fe VII], and both peaks have nearly the same heights in the other lines: the central peak at the velocity of about $+15 \mathrm{~km} \mathrm{~s}^{-1}$ is higher than the next redward peak in [Fe VII] and He II, while the opposite ratio is seen in the other lines.

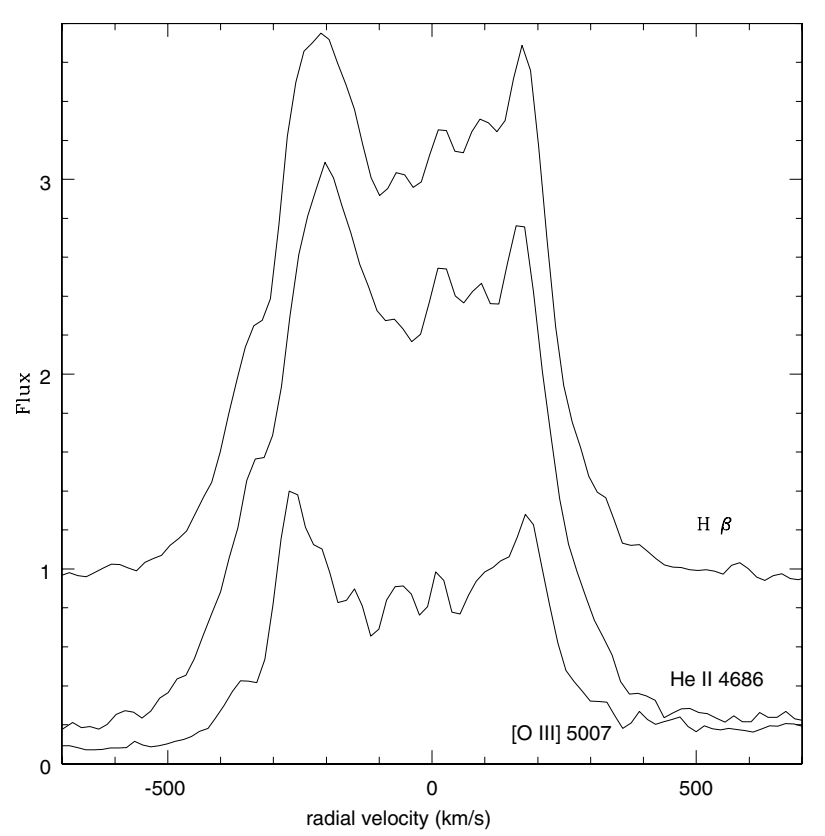

Fig. 24. Profiles of $\mathrm{H} \beta$, He II 4686, and [O III] 5007 on December 27, 1998. The ordinate is an intensity scale.

These results suggest that the shape of the nebulosity varied with different ionization states.

We are not able to make a quantitative argument for the intensities of the blue-shifted emission wings, because it is hard to measure their precise intensities. It seems that their intensities relative to those of the main emission bodies vary one by one. For example, the intensities of the wings of [N II] and [O III] lines are relatively low, while that of [Fe VII] is rather high. The emitting regions of the wings seem to have been different from those of the main emission bodies in the physical condition. The absence of the red-shifted wings suggests that the blue-shifted wings have been emitted in an asymmetric jet or outflow. The blue-shifts of the weak emission wings from the center of the main emission bodies are about $-300 \mathrm{~km} \mathrm{~s}^{-1}$ in $\mathrm{H} \mathrm{I}, \mathrm{He}$ II, and [Fe VII], and $-330 \mathrm{~km} \mathrm{~s}^{-1}$ in He I, [O III], and [N II].

\section{Properties of ejecta and the hot component}

As mentioned in the previous sections, the nebulosity of this nova probably became optically thin for H I lines in 1998. Therefore, we analyze the properties of the nebulosity using the spectra taken in and later than 1998.

\subsection{Electron temperature and density}

In the work of Nova (V1494) Aql 1999-II (Paper II), the electron temperature and density were derived using the intensity ratios of the nebular lines to the auroral lines of [O III] and [N II]. This method requires uniform electron temperature and density in the emitting regions of [O III] and [N II] lines. The same method is not used in this work, because the electron density in the emitting region of [N II] seems to have been different from that of [O III] region. The intensity ratios of 


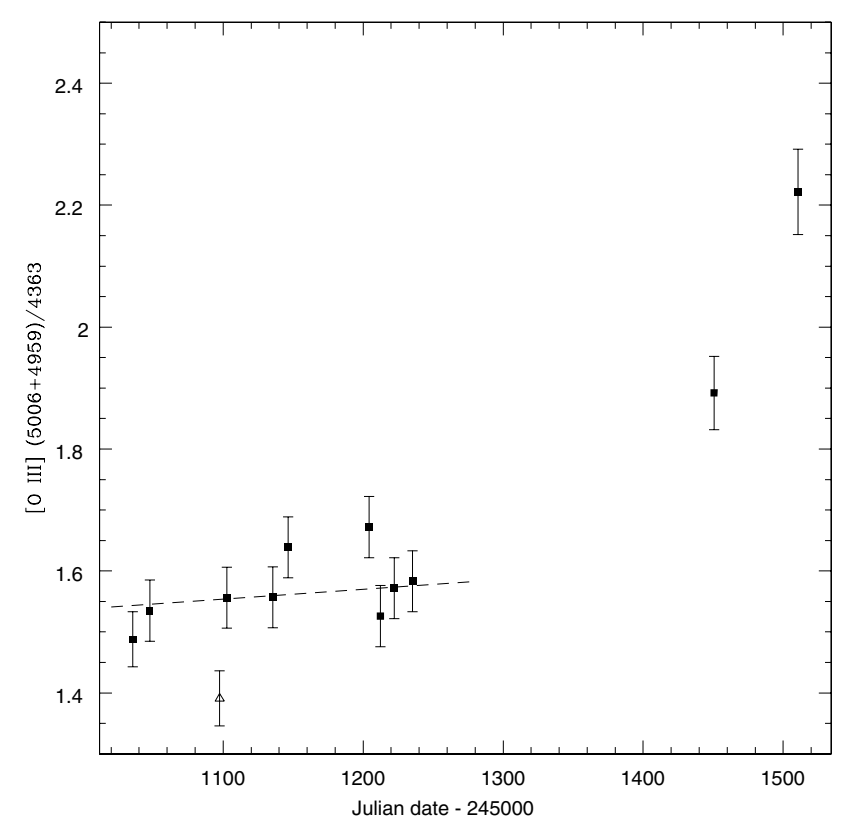

Fig. 25. Intensity ratio of the nebular to auroral lines of [O III]. The broken line shows the best-fitted line for the data until JD 2451235 (February 1999) except for the point indicated by an open triangle.

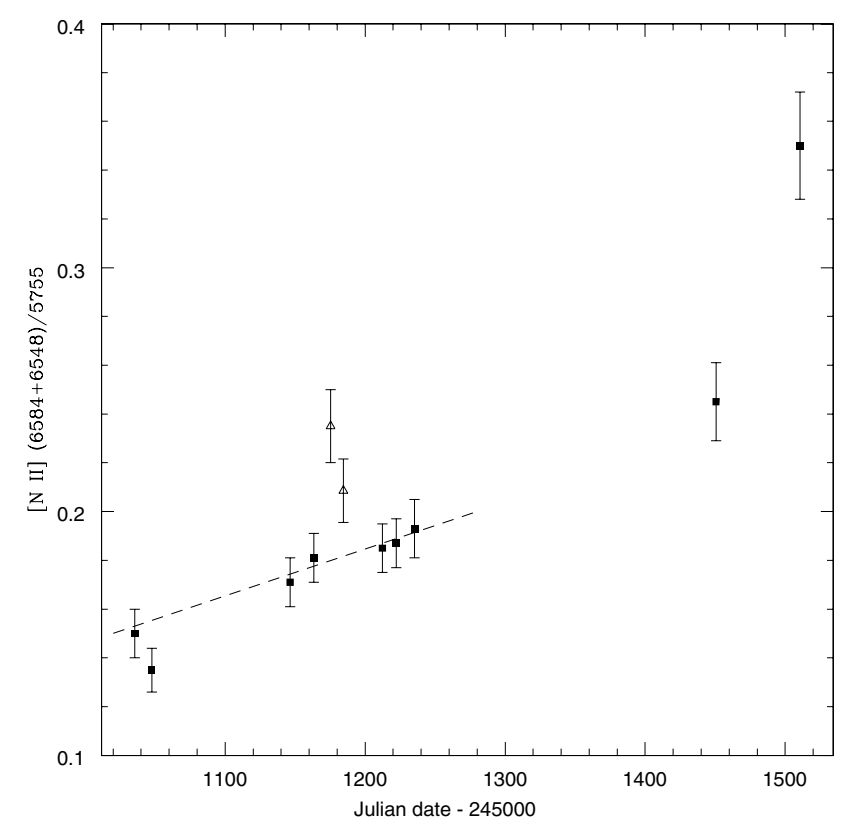

Fig. 26. Intensity ratio of the nebular to auroral lines of $[\mathrm{N} \mathrm{II].} \mathrm{The}$ broken line shows the best-fitted line for the data until JD 2451235 (February 1999) except for the points indicated by an open triangle.

the nebular lines to the auroral lines of [O III] and [N II] are shown in Figs. 25 and 26. As seen in the figures, the ratio of [O III] was nearly stable in the period between August 1998 and February 1999 (JD 2451 035-2 451 235), while a clear increase was seen in the ratio of $[\mathrm{N} \mathrm{II}]$ lines. It seems that the electron density in the $[\mathrm{N}$ II] region decreased more rapidly than in the [O III] region.

The low intensity ratios of [O III] lines observed until February 1999 (Fig. 25) suggest that the electron density in the emitting region of [O III] was still very high. Using the formula of Kaplan \& Pikelner (1970) for the [O III] line ratio in high density nebula $\left(N_{\mathrm{e}} \gg 2.5 \times 10^{7} \mathrm{~cm}^{-3}\right)$, we have an electron temperature $T_{\mathrm{e}}=10900 \pm 100 \mathrm{~K}$ from the line ratios observed until February 1999. On the other hand, the task NEBULAR.TEMDEN of IRAF gives a slightly higher temperature $T_{\mathrm{e}}=11000 \pm 60 \mathrm{~K}$ for the same line ratios at $N_{\mathrm{e}}=10^{8} \mathrm{~cm}^{-3}$. The latter electron temperature $T_{\mathrm{e}}=11000 \mathrm{~K}$ is used for the emitting regions of [O III] and [N II] lines. We assume that the electron temperature was stable until February 1999, because it is known that the electron temperatures of the ejecta of normal novae were nearly stable in the early nebular stage, as seen e.g. in V1500 Cyg (Ferland 1978; Lance et al. 1988), QU Vul (Saizar et al. 1992), and V1494 Aql (Paper II). The electron densities of the [O III] and [N II] regions were derived using the formulae of Seaton (1975) for the intensity ratios of the nebular to the auroral lines.

Unfortunately, it is difficult to estimate the precise intensities of [N II] $\lambda \lambda 6548$ and 6584 , because [N II] $\lambda 6584$ is badly blended with $\mathrm{H} \alpha$ and because [N II] $\lambda 6548$ is embedded in $\mathrm{H} \alpha$. The intensities of [N II] $\lambda 6584$ are obtained using a method for the deconvolution of blended lines described in Paper II, and the intensities of [N II] $\lambda 6548$ are derived assuming the theoretical ratio $I(6584) / I(6548)=2.94$ (Nussbaumer \& Rusca 1979). The errors in the intensities of [N II] $\lambda \lambda 6548$ and 6584, however, seem to be much larger than $10 \%$.

The dispersions of the observed line ratios in the period until JD 2451235 (Figs. 25, 26) may have been due mainly to observational errors. Therefore, we have made linear fittings for the ratios in the period from August 1998 to February 1999. The best-fitted lines are:

$$
\begin{aligned}
& \frac{I(5007+4959)}{I(4363)}=1.64 \times 10^{-4}(\mathrm{JD}-2451000)+1.54 \\
& \frac{I(6584+6548)}{I(5755)}=1.92 \times 10^{-4}(\mathrm{JD}-2451000)+0.146,
\end{aligned}
$$

which are shown by broken lines in the figures. The points indicated by an open triangle are not included in the fittings. The cause of such very large dispersions of those data is not known.

The electron densities, $N_{\mathrm{e}}$ (fit), of the [O III] and [N II] emitting regions are given in Table 9, which are derived with the intensity ratios expected from the best-fitted lines on their respective dates. These electron densities are used in the arguments that follow.

The electron density in the [O III] region decreased by $N_{\mathrm{e}}(\mathrm{fit}) \propto t^{-0.52}$, where $t$ is the number of days from maximum luminosity. The electron density in the $[\mathrm{N} \mathrm{II}]$ region decreased more rapidly, $N_{\mathrm{e}}(\mathrm{fit}) \propto t^{-4.3}$, which is faster than that expected from a spherical free expansion. This extremely high decline rate might not have been real. If the electron temperature in the emitting region of [N II] lines decreased during the period, we have a lower decline rate. It is, however, difficult to make a detailed argument about this phenomenon with our present material.

\subsection{Helium abundance}

As seen in the previous subsection, the electron density of the ejecta was still high even in the nebular stage. The collisional 
Table 9. Physical properties of ejecta and exciting star.

\begin{tabular}{|c|c|c|c|c|c|c|c|c|c|}
\hline Date & $\mathrm{JD}^{1}$ & $\begin{array}{l}N_{\mathrm{e}}(\mathrm{fit}) \\
{[\mathrm{O} \mathrm{III}]}\end{array}$ & $\begin{array}{l}N_{\mathrm{e}}(\mathrm{fit}) \\
{[\mathrm{N} \mathrm{II}]}\end{array}$ & $N\left(\mathrm{He}^{0}\right)$ & $N\left(\mathrm{He}^{+}\right)$ & $N(\mathrm{He})$ & $\begin{array}{c}m_{\mathrm{H}} \\
10^{-6} M_{\odot}\end{array}$ & $\begin{array}{c}m_{\text {total }} \\
10^{-6} M_{\odot}\end{array}$ & $\begin{array}{c}T_{*}^{2} \\
10^{4} \mathrm{~K}\end{array}$ \\
\hline \multicolumn{10}{|l|}{1998} \\
\hline Aug. 10 & 1035.6 & 73.1 & 72.4 & 0.097 & 0.090 & 0.187 & 3.1 & 4.2 & 19.2 \\
\hline Aug. 22 & 1047.6 & 72.6 & 67.4 & 0.090 & 0.095 & 0.185 & 3.5 & 4.8 & 19.6 \\
\hline Oct. 11 & 1097.6 & 70.9 & 52.2 & 0.112 : & 0.105 & $0.217:$ & & & \\
\hline Oct. 16 & 1102.7 & 70.7 & 51.0 & 0.090 & 0.101 & 0.191 & 3.0 & 4.2 & 20.1 \\
\hline Nov. 17 & 1135.4 & 69.5 & 44.6 & 0.078: & 0.102 & 0.180: & 2.9 & 3.9 & 20.3 \\
\hline Nov. 28 & 1146.4 & 69.2 & 42.8 & 0.081 & 0.097 & 0.178 & & & 19.9 \\
\hline Dec. 15 & 1163.5 & 68.6 & 40.2 & 0.075 & 0.096 & 0.171 & & & 19.8 \\
\hline Dec. 27 & 1175.4 & 68.2 & 38.6 & 0.074 & 0.096 & 0.170 & & & 19.9 \\
\hline \multicolumn{10}{|l|}{1999} \\
\hline Jan. 5 & 1184.4 & 67.9 & 37.5 & 0.073 & 0.107 & 0.181 & 2.5 & 3.4 & 20.7 \\
\hline Jan. 25 & 1204.3 & 67.2 & 35.3 & 0.088: & 0.110 & $0.197:$ & 2.6 & 3.5 & 20.8 \\
\hline Feb. $\quad 2$ & 1212.3 & 67.1 & 34.4 & 0.074 & 0.100 & 0.174 & & & 20.1 \\
\hline Feb. $\quad 12$ & 1222.3 & 66.7 & 33.4 & 0.082 & 0.106 & 0.188 & & & 20.6 \\
\hline Feb. 25 & 1235.3 & 66.3 & 32.2 & 0.070 & 0.106 & 0.177 & & & 20.7 \\
\hline
\end{tabular}

$\mathrm{JD}^{1}$ : Julian date $-2450000, N_{\mathrm{e}}$ (fit): electron density in $10^{6} \mathrm{~cm}^{-3}$ (see Sect. 7.1).

$T_{*}^{2}$ : Upper limit of the temperature of the exciting source.

excitation of He I lines cannot be neglected in such conditions (Clegg 1987; Peimbert \& Torres-Peimbert 1987). In this paper, we use the formulae of Clegg (1987) to estimate the ratios of collision/recombination of He I lines. The $\mathrm{C} / \mathrm{R}$ ratios at $T_{\mathrm{e}}=$ $11000 \mathrm{~K}$ and $N_{\mathrm{e}}=10^{8} \mathrm{~cm}^{-3}$ are 0.10 for He I $\lambda 4471,0.26$ for He I $\lambda 5876$, and 0.11 for He I $\lambda 6678$.

The method presented in Paper II was used to derive the helium abundance. The effective recombination coefficients of respective lines at $T_{\mathrm{e}}=11000 \mathrm{~K}$ are:

$\begin{array}{lll}\alpha_{\mathrm{H} \beta}^{\text {eff }} & =2.85 \times 10^{-14} & \mathrm{~cm}^{3} \mathrm{~s}^{-1} \\ \alpha_{\mathrm{HeI} 4471}^{\text {eff }} & =1.26 \times 10^{-14} & \mathrm{~cm}^{3} \mathrm{~s}^{-1} \\ \alpha_{\mathrm{He} \text { I5876 }}^{\text {eff }} & =4.42 \times 10^{-14} & \mathrm{~cm}^{3} \mathrm{~s}^{-1} \\ \alpha_{\mathrm{HeI} 6678}^{\text {eff }} & =1.42 \times 10^{-14} & \mathrm{~cm}^{3} \mathrm{~s}^{-1} \\ \alpha_{\mathrm{He} \mathrm{II} 4686}^{\text {eff }} & =3.00 \times 10^{-13} & \mathrm{~cm}^{3} \mathrm{~s}^{-1} .\end{array}$

These values were obtained making interpolations for the results of Brocklehurst (1972) and Hummer \& Storey (1987). The ionic abundances of helium have been estimated from the intensities of the helium lines relative to $\mathrm{H} \beta$ by using following formulae:

$$
\begin{aligned}
\frac{N\left(\mathrm{He}^{0}\right)}{N(\mathrm{H})} & =0.78 \frac{I(\mathrm{He} I 5876)}{I(\mathrm{H} \beta)} \\
& =2.08 \frac{I(\mathrm{He} \mathrm{I} 4471)}{I(\mathrm{H} \beta)} \\
& =2.75 \frac{I(\mathrm{He} \mathrm{I} 6678)}{I(\mathrm{H} \beta)} \\
\frac{N\left(\mathrm{He}^{+}\right)}{N(\mathrm{H})} & =0.092 \frac{I(\mathrm{He} \mathrm{II} \mathrm{4686)}}{I(\mathrm{H} \beta)} .
\end{aligned}
$$

The derived helium abundances are given in Table 9, where $N\left(\mathrm{He}^{0}\right)$ is a simple average of the abundances obtained by all available He I lines on each spectrum. The errors in the individual values are about \pm 0.02 and the values of lower accuracy are denoted by a colon. By taking an average, we have a helium abundance by atomic number $N(\mathrm{He}) / N(\mathrm{H})=0.18 \pm 0.01$ for this nova.

\subsection{Mass of ejecta}

The mass of the ejecta is estimated from the absolute intensity of $\mathrm{H} \beta$ emission line. The method follows the argument of Osterbrock (1989) and the formulae are given in Paper II. Using the effective recombination coefficient of $\mathrm{H} \beta$ given in the previous subsection, we have:

$m(\mathrm{H}) / M_{\odot}=\frac{D^{2}}{N_{\mathrm{e}}} 0.86 \times 10^{12} I(\mathrm{H} \beta)$,

in the case of $T_{\mathrm{e}}=11000 \mathrm{~K}$ and $N_{\mathrm{e}} \cong 10^{8} \mathrm{~cm}^{-3}$. In this equation, $m(\mathrm{H})$ is the mass of hydrogen in the nebula, $D$ the distance to the object in kpc, $N_{\mathrm{e}}$ the electron density in number $\mathrm{cm}^{-3}$, and $I(\mathrm{H} \beta)$ the intensity of the $\mathrm{H} \beta$ emission line in $\mathrm{erg} \mathrm{cm}^{-2} \mathrm{~s}^{-1}$.

The mass of helium in the ejecta is evaluated using the formulae given in Paper II. The total masses of the ejecta are given in Table 9. The apparent decrease in the mass may have been due to a decrease in the number of ionizing photons. The total mass of the ejecta is estimated at $5 \times 10^{-6} M_{\odot}$. This result, however, is only a very rough estimate because of the large ambiguity in the assumed distance $2.8 \mathrm{kpc}$. 
Table 10. Ionic abundances of heavy elements relative to $\mathrm{H}^{+}$.

\begin{tabular}{|c|c|c|c|c|c|c|c|c|}
\hline Date & $N^{+}$ & $\mathrm{O}^{++}$ & $\mathrm{Ne}^{++}$ & $\mathrm{Ne}^{+3}$ & $\mathrm{Ne}^{+4}$ & $\mathrm{Ar}^{++}$ & $\mathrm{Ar}^{+3}$ & $\mathrm{Ar}^{+4}$ \\
\hline \multicolumn{9}{|l|}{1998} \\
\hline Aug. 10 & 2.1 & 8.1 & & 8.8 & & 0.033 & 0.22 & 0.084 \\
\hline Aug. 22 & 2.2 & 9.4 & 8.1: & 9.1 & & & & 0.066 \\
\hline Oct. 11 & & 8.6 & & 6.9 & & & & \\
\hline Oct. 16 & 1.6 & 8.0 & 7.1 & 9.3 & 17.3 & & & 0.082 \\
\hline Nov. 17 & & 8.9 & 6.6 & 8.1 & & & & \\
\hline Nov. 28 & 1.4 & 8.0 & & 9.5 & & & & 0.085 \\
\hline Dec. 15 & 1.6 & 8.6 & & 8.5 & & 0.038 & 0.37 & 0.084 \\
\hline Dec. 27 & 1.4 & 8.7 & & 9.9 & & & & 0.089 \\
\hline \multicolumn{9}{|l|}{1999} \\
\hline Jan. 5,6 & 1.1 & 8.1 & & 7.5 & & & & 0.075 \\
\hline Jan. 25 & & 8.4 & & 8.6 & & & & \\
\hline Feb. $\quad 2$ & 1.2 & 7.2 & & 10.2 & & 0.057 & 0.24 & 0.088 \\
\hline Feb. $\quad 12$ & 1.2 & 7.6 & & 8.4 & & & & 0.10 \\
\hline Feb. 25 & 1.2 & 7.5 & & 10.7 & & 0.050 & & 0.10 \\
\hline
\end{tabular}

Unit of ionic abundances relative to $\mathrm{H}^{+}$is $10^{-4}$.

\subsection{Temperature of the hot component}

The intensities of the emission lines of He II 4686 and $\mathrm{H} \beta$ were roughly comparable in the nebular stage. It is possible to estimate the temperature of the ionizing source of such a nebula with the simple approximate formulae of Iijima (1981). This method is based on the following assumptions: the UV flux of the ionizing source is represented by one black-body function; all photons in the range $13.6-24.6 \mathrm{eV}$ are absorbed by hydrogen atoms; all photons in the range $24.6-54.4 \mathrm{eV}$ are absorbed by helium atoms; and all photons with energy higher than 54.4 $\mathrm{eV}$ are absorbed by singly ionized helium ions. When the electron temperature in the nebula is about $11000 \mathrm{~K}$, we have:

$$
\begin{aligned}
& T_{\star}\left(10^{4} \mathrm{~K}\right)=19.38 \mathrm{~K}^{1 / 2}+5.13 \\
& K=\frac{2.41 \cdot I(\mathrm{HeII} 4686) / I(\mathrm{H} \beta)}{4.42+9.9 \cdot I(\mathrm{HeI} 4471) / I(\mathrm{H} \beta)},
\end{aligned}
$$

where $I(\mathrm{He}$ II 4868), $I(\mathrm{H} \beta)$, and $I(\mathrm{He} \mathrm{I} 4471)$ are the intensities of their respective lines. The term $9.9 \cdot I(\mathrm{He}$ I 4471) can be replaced by either $3.7 \cdot I(\mathrm{He}$ I 5876) or $11.8 \cdot I(\mathrm{He}$ I 6678). If there is no emission line of [O I] on the spectra, as in the present case, this method gives an upper limit of the temperature (Iijima 1981). The derived upper limits of the temperature of the ionizing source are given in Table 9.

\subsection{Abundances of heavy elements}

Ionic abundances of nitrogen, oxygen, neon, and argon are derived using the task NEBULAR.IONIC of IRAF (Shaw \& Dufour 1995). The emission lines used in the estimates of the abundances are [N II] $\lambda \lambda 5755,6584$; [O III] $\lambda \lambda 4363,4959$, 5007; [Ne III] $\lambda 3869$; [Ne IV] $\lambda \lambda 4724,4725$; [Ne V] $\lambda 3426$; [Ar III] $\lambda 5192$; [Ar IV] $\lambda 4740$; and [Ar V] $\lambda \lambda 6435$ and 7006. The emission line at $\lambda 3727$ is not used for [O II], because it is too intense with respect to [O III] lines. If the line at $\lambda 3727$ were due to the blend of [O II] $\lambda \lambda 3726$ and 3729 , the ionic abundance $\mathrm{N}\left(\mathrm{O}^{+}\right)$would be ten times higher than $\mathrm{N}\left(\mathrm{O}^{++}\right)$derived by [O III] lines on the same spectra. This result conflicts with the highly ionized condition of the nebulosity and is also inconsistent with the absence of [O I] lines. As mentioned in Sect. 6.3, there may have been one more element in the emission line at $\lambda 3727 \AA$.

The task IONIC of IRAF requires electron temperature and density to obtain the abundances. The electron density derived from the ratios of [N II] lines is used only for [N II] lines and the one derived from [O III] lines is used for the other lines. The electron temperature $11000 \mathrm{~K}$ is assumed for all lines. When two or more lines are available for one ion, an average weighted by their intensities is taken. The ionic abundances relative to $\mathrm{H} \mathrm{I}$ in the unit of $10^{-4}$ are given in Table 10 . The abundance $N\left(\mathrm{Ne}^{+4}\right)$ on October 16, 1998 is rather uncertain, because the line [Ne V] $\lambda 3426$ is located near the blueward border of the spectrum where the error in the flux calibration is expected to be large.

It is difficult to estimate the atomic abundances of nitrogen and oxygen, because we have only $N\left(N^{+}\right)$for nitrogen and $N\left(\mathrm{O}^{++}\right)$for oxygen. Here, we make very rough estimates of the abundances of these atoms by making some simple assumptions:

$$
\begin{aligned}
\frac{N\left(\mathrm{O}^{++}\right)}{N\left(\mathrm{O}^{+}\right)} & =\frac{N\left(\mathrm{~N}^{++}\right)}{N\left(\mathrm{~N}^{+}\right)}=\frac{N\left(\mathrm{He}^{+}\right)}{N\left(\mathrm{He}^{0}\right)} \\
\frac{N\left(\mathrm{O}^{+3}+\mathrm{O}^{+4}\right)}{N\left(\mathrm{O}^{+}+\mathrm{O}^{++}\right)} & =\frac{N\left(\mathrm{~N}^{+3}+\mathrm{N}^{+4}\right)}{N\left(\mathrm{~N}^{+}+\mathrm{N}^{++}\right)} \\
& =\frac{N\left(\mathrm{Ne}^{+3}+\mathrm{Ne}^{+4}\right)}{N\left(\mathrm{Ne}^{+2}\right)}=3.7 \\
N\left(\mathrm{Ar}^{+6}\right) & =N\left(\mathrm{Ar}^{+5}\right)=N\left(\mathrm{Ar}^{+4}\right) .
\end{aligned}
$$

The low accuracy of $N\left(\mathrm{Ne}^{+4}\right)$ may not be a serious obstacle in such rough estimates. The ratios of the ionic abundances of neon on October 16, 1998 applies to all spectra. The 
Table 11. Chemical abundances of V723 Cas and other selected novae.

\begin{tabular}{lrrrrrr}
\hline \hline Element & V723 Cas & $\begin{array}{r}\text { RR Pic } \\
\text { Ref. 1 }\end{array}$ & HR Del & V1425 Aql & QU Vul & QU Vul \\
& & 2 & 3 & 4 & 5 \\
\hline$N(\mathrm{~N})$ & 9.2 & 9.70 & 9.54 & 10.18 & 9.72 & 9.05 \\
$N(\mathrm{O})$ & 9.8 & 8.78 & 9.68 & 10.02 & 9.53 & 9.27 \\
$N(\mathrm{Ne})$ & 9.5 & 9.00 & 8.38 & 8.66 & 9.58 & 9.41 \\
$N(\mathrm{Ar})$ & 7.7 & & & 6.71 & 6.42 & 6.04 \\
$N(\mathrm{He})$ & 11.26 & 11.36 & 11.43 & 11.12 & 11.70 & 11.06 \\
\hline
\end{tabular}

The abundances are given by atomic number in logarithmic scale relative to hydrogen $N(\mathrm{H})=12$.

Ref. 1: Williams \& Gallagher (1979), 2: Tylenda (1978), 3: Lyke et al. (2001), 4: Saizar et al. (1992), 5: Schwarz (2002).

abundances of ions higher than $N^{+4}, \mathrm{O}^{+4}, \mathrm{Ne}^{+5}$, and $\mathrm{Ar}^{+6}$ are omitted.

The derived abundances are given in Table 11 on a logarithmic scale relative to hydrogen $N(\mathrm{H})=12$. The abundances of a fast nova V1425 Aql (Lyke et al. 2001), a slow nova QU Vul (Saizar et al. 1992; Schwarz 2002), and the extremely slow novae HR Del (Tylenda 1978) and RR Pic (Williams \& Gallagher 1979) are also given in the table.

The abundance of nitrogen is lowest among those of the novae in the table except for the new result of QU Vul by Schwarz (2002). The unusually low abundance of oxygen of RR Pic does not occur in this nova. The weakness of [O III] lines in the nebular stage may have been due mainly to the high density in the ejecta. The abundance of neon is comparable to that of QU Vul and higher than those of the other novae, while QU Vul is classified as a neon nova; that is, its explosion is expected to have occurred on an O-Ne-Mg white dwarf (Saizar et al. 1992; Schwarz 2002). The low abundance of argon of QU Vul, however, is not found in this nova.

\section{Discussion and summary}

An extremely slow spectral evolution is the most notable characteristic of V723 Cas. The interval from maximum luminosity to the beginning of nebular stage is about 18 months, which is much longer than the 10 months of RR Pic or 8 months of HR Del. The nova RR Pic had been known as the slowest until the appearance of this object and HR Del was the second.

Some symbiotic novae, e.g., RR Tel (Thackeray 1977) and PU Vul (Iijima 1989), showed still slower spectral evolutions. Some spectral features of V723 Cas, e.g., the emission line at $\lambda 3727 \AA$, are also similar to those of symbiotic novae as shown in Sect. 6. We do not, however, support the classification of V723 Cas as a symbiotic nova as suggested by Munari et al. (1996). Symbiotic novae are believed to be long-period binary systems consisting of red giants and hot components. On the contrary, V723 Cas is a short-period binary system (Goranskij et al. 2000), and there is no trace of a red giant in the infrared photometry (Kamath \& Ashok 1999). The three objects, V723 Cas, RR Pic, and HR Del, seem to constitute a group of rare classical novae of extremely slow spectral evolutions.

Friedjung (1992) studied the long pre-maximum stage of HR Del and suggested that its outburst occurred under a marginal condition of thermo-nuclear runaway on a white dwarf. It has been known that HR Del and V723 Cas are very similar in their properties of the long pre-maximum stages (e.g., Paper I; Friedjung \& Iijima 2002). Hachisu \& Kato (2004) have recently made theoretical model calculations of the outbursts of slow novae, and find that a model of thermonuclear runaway on a less massive $\left(0.59 M_{\odot}\right)$ white dwarf with a massive hydrogen rich envelope best represents the light curve of V723 Cas, including its long pre-maximum stage. As mentioned in Sect. 3, the distance derived in their model agrees well with our estimate. On the other hand, the wind velocity expected in their model is about $500 \mathrm{~km} \mathrm{~s}^{-1}$ (Hachisu \& Kato 2004), while the maximum outflow velocity observed in this nova is about $1600 \mathrm{~km} \mathrm{~s}^{-1}$. It seems that some discrepancies are still left between the theory and the observations.

The main aim of this paper is to present the basic spectroscopic material of the slowest nova V723 Cas. We hope that our results will be useful in advancing research on the outbursts of novae under a marginal condition.

Acknowledgements. We are grateful to Profs. I. Hachisu and M. Kato for the useful discussions and comments, and to Prof. R. Barbon for the careful reading of the manuscript and valuable suggestions. Thanks are also due to Dr. T. Kato, Dr. D. Nogami, Mr. S. Kiyota, and the many other professional and amateur astronomers who supplied photometric data of V723 Cas on the VS-NET.

\section{References}

Brocklehurst, M. 1972, MNRAS, 157, 211

Cardelli, J. A., Clayton, G. C., \& Mathis, J. S. 1989, ApJ, 345, 245

Chochol, D., \& Pribulla, T. 1977, Contrib. Astron. Obs. Skalnatè Pleso, 27, 53

Clegg, R. E. S. 1987, MNRAS, 229, 31P

Della Valle, M., \& Livio, M. 1995, ApJ, 452, 704

Evans, A., Gehrz, R. D., \& Geballe, T. R. 2003, AJ, 126, 1981

Ferland, G. J. 1978, ApJ, 219, 589

Ferland, G., Lambert, D. L., \& Woodman, J. H. 1977, ApJ, 213, 132

Friedjung, M. 1992, A\&A, 262, 487

Friedjung, M., \& Iijima, T. 2002, in Classical Nova Explosions, ed. M. Hernanz, \& J. Josè, AIP Conf. Proc., 637, 308

Gonzalez-Riestra, R., Shore, S. N., Starrfield, S., \& Krautter, J. 1996, IAU Circ., 6295

Goranskij, V. P., Shugarov, S. Y., Katysheva, N. A., et al. 2000, IBVS, 4852

Greenhouse, M. A., Grasdalen, G. L., Hayward, T. L., Gehrz, R. D., \& Janes, T. J. 1988, AJ, 95, 172

Hachisu, I., \& Kato, M. 2004, ApJ, 612, L57

Hummer, D. G., \& Storey, P. J. 1987, MNRAS, 224, 801 
Iijima, T. 1981, in Photometric and Spectroscopic Binary Systems, ed.

E. D. Carling, \& Z. Kopal (D. Reidel Publ. Co.), 517

Iijima, T. 1989, A\&A, 215, 57

Iijima, T., \& Rosino, L. 1997, IAU Circ., 6703

Iijima, T., \& Esenoglu, H. H. 2003, A\&A, 404, 997 (Paper II)

Iijima, T., Rosino, L., \& Della Valle, M. 1998, A\&A, 338, 1006 (Paper I)

Jones, H. S. 1931, MNRAS, 91, 777

Kamath, U. S., \& Ashok, N. M. 1999, A\&AS, 136, 107

Kaplan, S. A., \& Pikelner, S. B. 1970, The Interstellar Medium (Harvard Univ. Press, Cambridge, MA), 107

Krautter, J., \& Williams, R. E. 1989, ApJ, 341, 968

Lance, C. M., McCall, M. L., \& Uomoto, A. K. 1988, ApJS, 66, 151

Lyke, J. E., Gehrz, R. D., Woodward, C. E., et al. 2001, AJ, 122, 3305

McLaughlin, D. B. 1953, ApJ, 118, 27

McLaughlin, D. B. 1960, in Stellar Atmospheres, ed. J. L. Greenstein (The University of Chicago Press), 585

Meinel, A. B., Aveni, A. F., \& Stockton, M. W. 1968, Catalog of Emission Lines in Astrophysical Objects, Optical Sciences Center and Steward Obs., Univ. of Arizona, Tucson

Moore, C. E. 1959, A Multiplet Table of Astrophysical Interest, US Dept. of Commerce, Office of Technical Services, Washington DC

Munari, U., Goranskij, V. P., Popova, A. A., et al. 1996, A\&A, 315, 166

Netzer, H. 1975, MNRAS, 171, 395
Nussbaumer, H., \& Rusca, C. 1979, A\&A, 72, 129

Ohshima, O., Akazawa, H., \& Ohkura, N. 1996, IBVS, 4295

Osterbrock, D. E. 1989, Astrophysics of Gaseous Nebulae and Active Galactic Nuclei (Mill Valley, CA: University Science Books)

Peimbert, M., \& Torres-Peimbert, S. 1987, Rev. Mex. Astron Astrofis., 14,540

Pèquignot, D., Petitjean, P., Boisson, C., \& Krautter, J. 1993, A\&A, 271, 219

Rafanelli, P., \& Rosino, L. 1978, A\&AS, 31337

Rudy, R. J., Venturini, C. C., Lynch, D. K., Mazuk, S., \& Puetter, R. C. 2002, ApJ, 573, 794

Saizar, P., Starrfield, S., Ferland, G. J., et al. 1992, ApJ, 398, 651

Schmid, H. M., \& Schild, H. 1990, MNRAS, 246, 84

Schwarz, G. J. 2002, ApJ, 577, 940

Seaton, M. J. 1975, MNRAS, 170, 475

Shaw, R. A., \& Dufour, R. J. 1995, PASP, 107, 896

Thackeray, A. D. 1977, Mem. R. Astron. Soc., 83, 1

Tylenda, R. 1978, Acta Astron., 28, 333

Warner, B. 1995, Cataclysmic Variable Stars (Cambridge Univ. Press) Williams, R. E., \& Gallagher, J. S. 1979, ApJ, 228, 482

Williams, R. E., Hamuy, M., Phillips, M. M., et al. 1991, ApJ, 376, 721

Yamamoto, M. 1995, IAU Circ., 6213

Zhu, Zh-X., \& Hang, H.-R. 1999, Acta Astron. Sinica, 40, 255 
T. Iijima: Spectral evolution of the slowest classical nova V723 Cassiopeiae in the decline stage, Online Material p 1

\section{Online Material}


T. Iijima: Spectral evolution of the slowest classical nova V723 Cassiopeiae in the decline stage, Online Material p 2

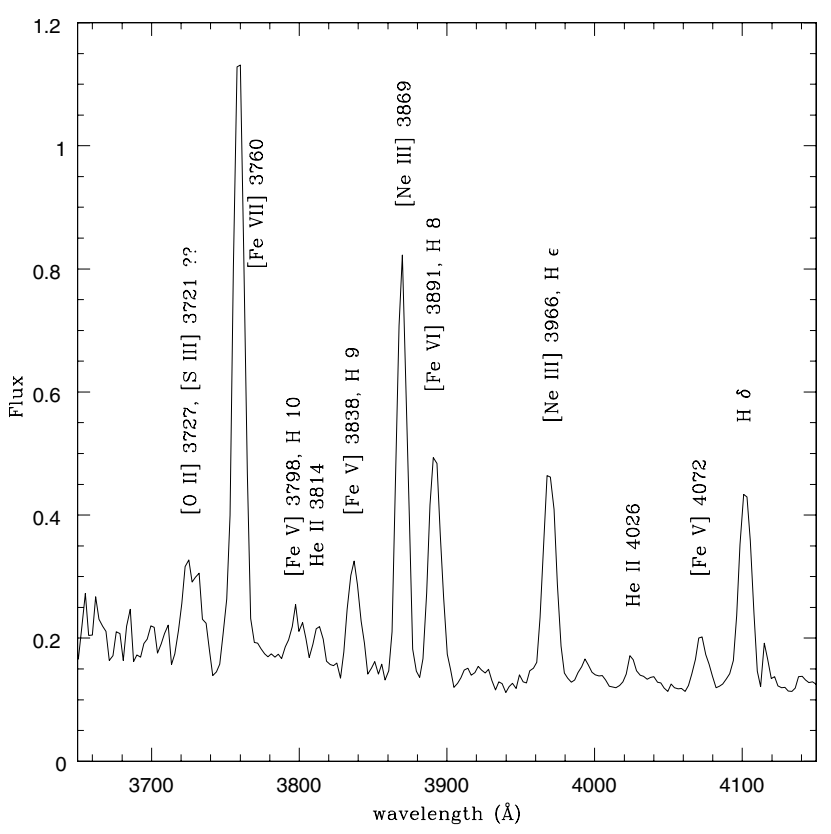

Fig. 21. A grating blue spectrum of V723 Cas on Nov. 28, 1999. The unit of ordinate is $10^{-13} \mathrm{erg} \mathrm{cm}^{-2} \mathrm{~s}^{-1} \AA^{-1}$.

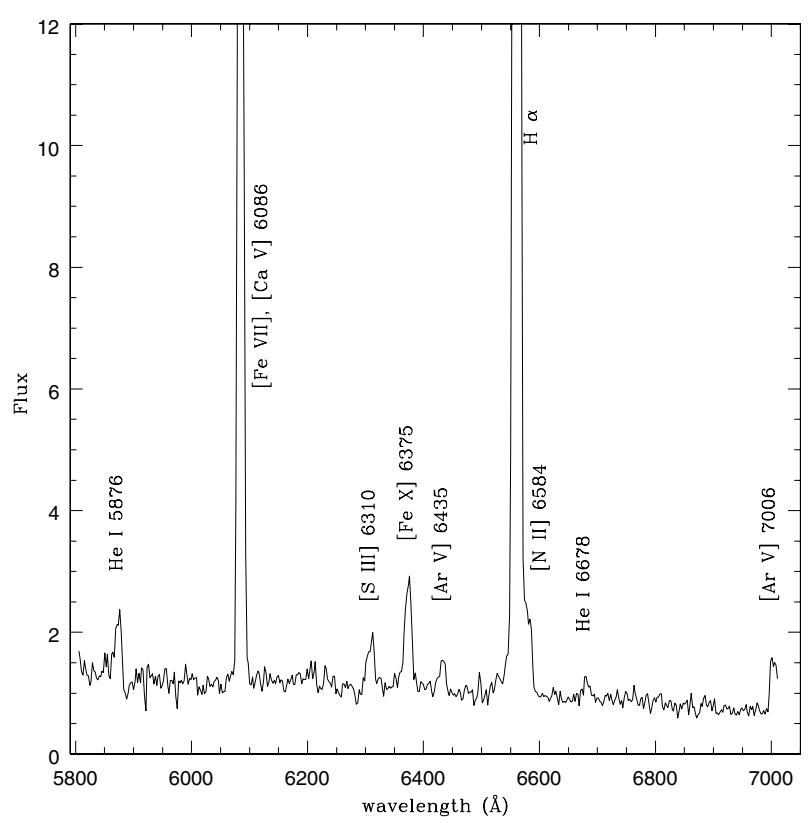

Fig. 22. A grating red spectrum of V723 Cas on Oct. 15, 2001. The unit of ordinate is $10^{-14} \mathrm{erg} \mathrm{cm}^{-2} \mathrm{~s}^{-1} \AA^{-1}$. 
T. Iijima: Spectral evolution of the slowest classical nova V723 Cassiopeiae in the decline stage, Online Material p 3

Table 1. Spectroscopic observations of V723 Cas in the decline stage.

\begin{tabular}{|c|c|c|c|c|c|c|}
\hline Date & $\mathrm{UT}^{1}$ & $\mathrm{JD}^{2}$ & Days $^{3}$ & $\begin{array}{r}\text { Exp. } \\
\text { s }\end{array}$ & Inst. & $\begin{array}{c}\text { Range } \\
\mathrm{nm}\end{array}$ \\
\hline \multicolumn{7}{|l|}{1996} \\
\hline Jan. 2 & $22: 15$ & 85.4 & 16.1 & 600 & $\mathrm{~B} \& \mathrm{C}$ & $360-470$ \\
\hline Jan. 2 & $23: 17$ & 85.5 & 16.2 & 300 & " & $462-572$ \\
\hline Jan. 2 & $23: 59$ & 85.5 & 16.2 & 60 & " & $567-680$ \\
\hline Jan. 15 & $18: 35$ & 98.3 & 29.0 & 300 & $\mathrm{C}-\mathrm{VI}$ & $412-510$ \\
\hline Jan. 16 & $18: 25$ & 99.3 & 30.0 & 300 & $"$ & $412-510$ \\
\hline Jan. 17 & $18: 04$ & 100.3 & 31.0 & 180 & " & $483-723$ \\
\hline Jan. 17 & $18: 53$ & 100.3 & 31.0 & 300 & " & $412-510$ \\
\hline Feb. $\quad 7$ & $18: 31$ & 121.3 & 52.0 & 600 & " & $412-510$ \\
\hline Feb. $\quad 7$ & 20:01 & 121.3 & 52.0 & 120 & " & $483-723$ \\
\hline Feb. 8 & $18: 39$ & 122.3 & 53.0 & 600 & " & $412-510$ \\
\hline Feb. 14 & $18: 48$ & 128.3 & 59.0 & 600 & " & $412-510$ \\
\hline Feb. $\quad 17$ & $19: 28$ & 131.3 & 62.0 & 600 & $"$ & $412-510$ \\
\hline Feb. 28 & $18: 46$ & 142.3 & 73.0 & 900 & " & $412-510$ \\
\hline March 1 & $18: 32$ & 144.3 & 75.0 & 900 & " & $412-510$ \\
\hline March 2 & $18: 45$ & 145.3 & 76.0 & 300 & $"$ & $483-723$ \\
\hline March 5 & 19:06 & 148.3 & 79.0 & 600 & " & $412-510$ \\
\hline March 14 & $18: 46$ & 157.3 & 88.0 & 900 & " & $412-510$ \\
\hline June 6 & $1: 56$ & 240.6 & 171.3 & 300 & $\mathrm{~B} \& \mathrm{C}$ & $395-508$ \\
\hline July 27 & $2: 40$ & 291.6 & 222.3 & 300 & Ech & $433-688$ \\
\hline $\begin{array}{c}\text { Dec. } 15 \\
1997\end{array}$ & $21: 42$ & 433.4 & 364.2 & 600 & C-VI & $457-630$ \\
\hline Jan. 18 & $19: 16$ & 467.3 & 398.0 & 1200 & C-VI & $412-510$ \\
\hline Jan. 28 & $19: 13$ & 477.3 & 408.0 & 2400 & Ech & $460-710$ \\
\hline Jan. 29 & $20: 19$ & 478.4 & 409.1 & 1200 & $"$ & $460-710$ \\
\hline Feb. $\quad 10$ & $18: 22$ & 490.3 & 421.0 & 1200 & $\mathrm{~B} \& \mathrm{C}$ & $395-508$ \\
\hline Feb. $\quad 10$ & $19: 38$ & 490.3 & 421.0 & 120 & $"$ & $572-683$ \\
\hline Feb. 20 & $18: 55$ & 500.3 & 431.0 & 600 & Ech & $460-710$ \\
\hline March 29 & $18: 56$ & 537.3 & 468.0 & 600 & " & $460-710$ \\
\hline May 19 & $2: 38$ & 587.6 & 518.3 & 180 & $\mathrm{C}-\mathrm{VI}$ & $412-510$ \\
\hline May 30 & $2: 24$ & 598.6 & 529.3 & 300 & Ech & $460-710$ \\
\hline July 1 & 2:00 & 630.6 & 561.3 & 900 & " & $460-710$ \\
\hline July $\quad 21$ & $2: 10$ & 650.6 & 581.3 & 1200 & $\mathrm{C}-\mathrm{VI}$ & $376-432$ \\
\hline July 22 & $1: 57$ & 651.6 & 582.3 & 1200 & $"$ & $412-510$ \\
\hline $\begin{array}{c}\text { Sept. } 23 \\
1998\end{array}$ & $1: 24$ & 714.6 & 645.3 & 1200 & " & $412-510$ \\
\hline Aug. 10 & $1: 55$ & 1035.6 & 966.3 & 2400 & Ech & $433-688$ \\
\hline Aug. 21 & $2: 58$ & 1046.6 & 977.3 & 1200 & $\mathrm{~B} \& \mathrm{C}$ & $569-686$ \\
\hline Aug. 22 & $2: 02$ & 1047.6 & 978.3 & 1800 & $"$ & $392-508$ \\
\hline Oct. 11 & $1: 52$ & 1097.6 & 1028.3 & 1800 & " & $400-516$ \\
\hline Oct. 16 & $1: 56$ & 1102.6 & 1033.3 & 2400 & " & $335-448$ \\
\hline Oct. 16 & $3: 36$ & 1102.7 & 1033.4 & 1200 & " & $433-550$ \\
\hline Oct. 16 & $4: 06$ & 1102.7 & 1033.4 & 600 & $"$ & $539-657$ \\
\hline Nov. 17 & $22: 07$ & 1135.4 & 1066.1 & 1800 & $"$ & $395-512$ \\
\hline Nov. 28 & $22: 26$ & 1146.4 & 1077.1 & 1800 & Ech & $433-688$ \\
\hline Dec. & $21: 59$ & 1163.5 & 1094.2 & 2400 & $" \prime$ & $460-710$ \\
\hline Dec. 27 & $20: 55$ & 1175.4 & 1106.1 & 2400 & $"$ & $433-688$ \\
\hline
\end{tabular}


T. Iijima: Spectral evolution of the slowest classical nova V723 Cassiopeiae in the decline stage, Online Material p 4

Table 1. continued.

\begin{tabular}{|c|c|c|c|c|c|c|}
\hline Date & $\mathrm{UT}^{1}$ & $\mathrm{JD}^{2}$ & Days $^{3}$ & $\begin{array}{r}\text { Exp. } \\
\mathrm{s}\end{array}$ & Inst. & $\begin{array}{c}\text { Range } \\
\mathrm{nm}\end{array}$ \\
\hline \multicolumn{7}{|l|}{1999} \\
\hline Jan. 5 & $22: 03$ & 1184.4 & 1115.1 & 1800 & $B \& C$ & $397-514$ \\
\hline Jan. & $23: 16$ & 1184.5 & 1115.2 & 1200 & " & $493-610$ \\
\hline Jan. & $19: 54$ & 1185.3 & 1116.0 & 1200 & " & $593-712$ \\
\hline Jan. & $21: 11$ & 1185.4 & 1116.1 & 1800 & " & $492-612$ \\
\hline Jan. 25 & 19:00 & 1204.3 & 1135.0 & 1800 & " & $404-521$ \\
\hline Feb. $\quad 2$ & $19: 46$ & 1212.3 & 1143.0 & 2400 & Ech & $433-688$ \\
\hline Feb. $\quad 12$ & $19: 36$ & 1222.3 & 1153.0 & 2400 & " & $433-688$ \\
\hline Feb. 25 & $19: 10$ & 1235.3 & 1166.0 & 3000 & " & $433-688$ \\
\hline Sept. 29 & 1:59 & 1450.6 & 1381.3 & 2400 & $"$ & $433-688$ \\
\hline Nov. 28 & $0: 42$ & 1510.6 & 1441.3 & 3600 & $B \& C$ & $394-512$ \\
\hline Nov. 28 & $1: 52$ & 1510.6 & 1441.3 & 1200 & " & 556-674 \\
\hline Nov. 28 & 23:01 & 1511.5 & 1442.2 & 3600 & " & $363-480$ \\
\hline Nov. 29 & $23: 58$ & 1512.5 & 1443.2 & 1800 & " & $500-618$ \\
\hline $\begin{array}{c}\text { Nov. } 30 \\
2000\end{array}$ & $1: 27$ & 1512.6 & 1443.3 & 1800 & " & 613-731 \\
\hline $\begin{array}{l}\text { Feb. } \quad 5 \\
2001\end{array}$ & 20:09 & 1580.4 & 1511.1 & 2400 & $B \& C$ & $581-700$ \\
\hline Feb. 11 & 20:00 & 1952.3 & 1883.0 & 1800 & $B \& C$ & $581-700$ \\
\hline Feb. & $19: 05$ & 1957.3 & 1888.0 & 2400 & " & $401-518$ \\
\hline Feb. $\quad 18$ & $19: 18$ & 1959.3 & 1890.0 & 1800 & $"$ & $588-707$ \\
\hline Sept. 27 & $1: 36$ & 2179.6 & 2110.3 & 3600 & " & $400-515$ \\
\hline Oct. 14 & $0: 42$ & 2196.6 & 2127.3 & 3600 & " & $400-515$ \\
\hline Oct. 15 & $2: 02$ & 2197.6 & 2128.3 & 2400 & " & $581-700$ \\
\hline Dec. & $21: 53$ & 2255.5 & 2186.2 & 2400 & " & $598-717$ \\
\hline
\end{tabular}

$\mathrm{UT}^{1}$ : Universal time at beginning of exposure. $\mathrm{JD}^{2}$ : Julian date -2450000.

Days $^{3}$ : Number of days from maximum (JD 2450 069.3). 
T. Iijima: Spectral evolution of the slowest classical nova V723 Cassiopeiae in the decline stage, Online Material p 5

Table 2. Intensities of emission lines relative to $\mathrm{H} \beta=100$ on January 2, 1996.

\begin{tabular}{|c|c|c|}
\hline $\begin{array}{l}\lambda_{\odot} \\
(\AA)\end{array}$ & $I(\lambda)$ & Identification \\
\hline 3798.2 & 10.2 & H 10 \\
\hline 3814.5 & 3.6 & Fe II $153,3814.1$ \\
\hline 3836.6 & 13.2 & H 9 \\
\hline 3889.6 & 16.5 & H 8 \\
\hline 3901.6 & 6.3 & Ti II 34, 3900.5 \\
\hline 3914.2 & 4.9 & Fe II 3, 3914.5 \\
\hline 3935.0 & 17.2 & Ca II 1, 3933.7, Fe II 173, -35.9 \\
\hline 3970.5 & 24.5 & $\mathrm{H} \epsilon, \mathrm{Ca}$ II $1,3968.5$ \\
\hline 4003.4 & 1.8 & Fe II 190, 29, 4002.5, -02.1 \\
\hline 4011.9 & 2.5 & Fe II 126, 4012.5 \\
\hline 4025.6 & 7.5 & Fe II 127, 4024.6, He I -26.2 \\
\hline 4033.5 & 1.1: & Fe II 126, 4032.9 \\
\hline 4052.4 & 3.5 & Ti II 87, 4053.8, Cr II 19, -54.1 \\
\hline 4064.4 & 3.9 & Fe II 39, 4064.8, unid -64.5 \\
\hline 4101.4 & 31.4 & $\mathrm{H} \delta$ \\
\hline 4120.4 & 3.5: & Fe II $28,4122.6$ \\
\hline 4126.3 & 4.5: & Si II, 4128.1 \\
\hline 4143.6 & 1.3 & He I, 4143.8 \\
\hline 4161.3 & 3.7 & Ti II 105, 4163.6 \\
\hline 4174.5 & 12.4 & Fe II 27, 4173.5, Fe II 28, -78.9 \\
\hline 4201.8 & 3.3 & {$[\mathrm{Ni}$ II] $3 \mathrm{~F}, 4201.7$} \\
\hline 4214.0 & 0.7 & Sr II $1,4215.5$ \\
\hline 4231.6 & 7.3 & Fe II 27, 4233.2 \\
\hline 4240.1 & 1.3 & N II 47, 4241.8 \\
\hline 4256.4 & 2.7 & Fe II $28,4258.2$ \\
\hline 4271.8 & 3.5 & Fe II $27,4273.3$ \\
\hline 4288.9: & 5.9: & Ti II 41, 4290.2 \\
\hline 4299.6 & 9.3 & Ti II 41, 4300.1 \\
\hline 4314.4 & 5.6 & Fe II 32, 4314.3, Ti II 41, -15.0 \\
\hline 4341.0 & 41.8 & $\mathrm{H} \gamma$ \\
\hline 4350.8 & 9.4: & Fe II $27,4351.8$ \\
\hline 4367.2 & 1.9 & Ti II 104, 4367.7 \\
\hline 4373.9 & 2.6 & {$[\mathrm{Fe}$ II] $21 \mathrm{~F}, 4372.4, \mathrm{Ti}$ II 93, -74.8} \\
\hline 4385.4 & 7.5 & Fe II $27,4385.4$ \\
\hline 4395.7 & 5.6 & Ti II 19, 4395.0 \\
\hline 4416.4 & 8.0 & Fe II 27, 4416.8, [Fe II] 6F, -16.3 \\
\hline 4443.5 & 3.1 & Ti II $19,4443.8$ \\
\hline 4469.6 & 9.7 & He I, 4471.6 \\
\hline 4489.5 & 8.5 & Fe II 37, 4489.2, -91.4 \\
\hline 4508.1 & 4.7: & Fe II $38,4508.3$ \\
\hline 4519.9 & 10.8 & Fe II 37, 4520.2 \\
\hline 4533.0 & 3.4 & {$[\mathrm{Fe} \mathrm{II}] 6 \mathrm{~F}, 4533.0$} \\
\hline 4551.0 & 5.6: & Fe II 38, 4549.5 \\
\hline 4555.9 & 4.9: & $\mathrm{Fe}$ II $37,4555.9$ \\
\hline 4574.8 & 3.6: & Fe II $38,4576.3$ \\
\hline 4583.2 & 10.9 & Fe II $38,4583.8$ \\
\hline 4618.6 & 4.8 & Fe II $38,4620.5$ \\
\hline 4629.5 & 8.4 & Fe II $37,4629.3$ \\
\hline
\end{tabular}


T. Iijima: Spectral evolution of the slowest classical nova V723 Cassiopeiae in the decline stage, Online Material p 6

Table 2. continued.

\begin{tabular}{|c|c|c|}
\hline $\begin{array}{l}\lambda_{\odot} \\
(\AA)\end{array}$ & $I(\lambda)$ & Identification \\
\hline 4656.3 & 2.0 & Fe II $43,4657.0$ \\
\hline 4666.4 & 4.8 & Fe II 37, 4666.8 \\
\hline 4683.3 & 1.7 & Si II 13, 4683.8 \\
\hline 4709.8 & 1.9 & He I, 4713.1 \\
\hline 4730.2 & 3.0 & Fe II $43,4731.4$ \\
\hline 4762.8 & 1.5 & Ti II 48, 4763.8 \\
\hline 4778.8 & 1.0 & N II $20,4779.7,-81.2$ \\
\hline 4803.9 & 1.8: & N II $20,4803.3$ \\
\hline 4823.5 & 2.4 & Cr II 30, 4824.1 \\
\hline 4860.8 & 100 & $\mathrm{H} \beta$ \\
\hline 4910.5 & 1.6 & Ti II 114, 4911.2 \\
\hline 4923.1 & 9.7 & He I, 4921.9, Fe II 42, -23.9 \\
\hline 4992.0 & 3.0: & Fe II 36, 4993.4 \\
\hline 5017.5 & 13.7 & He I, 5015.7, Fe II 42, -18.4 \\
\hline 5031.6 & 0.8 : & unid 5033.0 \\
\hline 5038.7 & 0.9: & Si II 5, 5041.1 \\
\hline 5051.7 & 1.4: & Si II 5, 5056.0 \\
\hline 5070.3 & 0.7 & [Fe II] 19F, 5072.4 \\
\hline 5098.9 & 1.0 & Fe II $185,5100.8$ \\
\hline 5107.8 & 0.5 : & [Fe II] $18 \mathrm{~F}, 5108.0$ \\
\hline 5129.3 & 2.9 & Ti II 86, 5129.1 \\
\hline 5153.8 & 2.0 & Fe II $35,5154.4$ \\
\hline 5168.8 & 11.0 & Fe II 42, 5169.0 \\
\hline 5185.0 & 3.3 & [Fe II] 19F, 5184.8 \\
\hline 5196.0 & 5.3 & Fe II 49, 5197.6 \\
\hline 5210.1 & 1.2: & Ti II 103, 5211.5 \\
\hline 5233.8 & 6.5 & Fe II 49, 5234.6 \\
\hline 5253.0: & 1.9: & Fe II 49, 5253.0 \\
\hline 5264.1: & 2.3: & Fe II $48,5264.8$ \\
\hline 5277.2 & 9.8 & Fe II $49,5276.0$ \\
\hline 5315.9 & 9.5 & Fe II 49, 5316.6 \\
\hline 5335.8 & 2.5 & Fe II $48,5337.7$ \\
\hline 5361.8 & 3.8 & Fe II $48,5362.9$ \\
\hline 5380.2 & 0.6 & Ti II 69, 5381.0 \\
\hline 5394.7 & 0.6 & Ti II 102, 5396.6 \\
\hline 5409.8 & 1.4 & [Fe II] 17F, 5412.6 \\
\hline 5424.3 & 3.3 & Fe II 49, 5425.3 \\
\hline 5477.1 & 0.8 & Fe II $49,5477.7$ \\
\hline 5490.0 & 0.6 & unid 5491.7 \\
\hline 5504.6 & 1.1 & Cr II 50, 5503.2, unid -05.0 \\
\hline 5532.6 & 4.7 & Fe II 55, 5534.9 \\
\hline 5566.7: & $0.4:$ & Fe II -, 5567.8 \\
\hline 5585.9 & 0.5 : & [Fe II] 39F, 5588.2 \\
\hline 5640.7 & 0.5 : & S II $11,5640.3$, Sc II $29,-41.0$ \\
\hline 5658.2 & 0.9 : & Sc II 29, 5657.9, Fe II 57, -57.9 \\
\hline 5668.6 & 0.6 : & Sc II 29, 5669.0 \\
\hline
\end{tabular}


T. Iijima: Spectral evolution of the slowest classical nova V723 Cassiopeiae in the decline stage, Online Material p 7

Table 2. continued.

\begin{tabular}{ccl}
\hline \hline$\lambda_{\odot}$ & $I(\lambda)$ & Identification \\
$(\AA)$ & & \\
\hline 5682.4 & $0.6:$ & Sc II 29, 5684.2, [Fe II] 33F, -83.6 \\
5748.0 & $0.3:$ & N II 9, 5747.3 \\
5810.5 & $0.4:$ & Ti II 79, 5814.6, Fe II 169, -13.7 \\
5822.7 & $0.3:$ & Fe II 58, 5824.4 \\
5832.3 & $0.4:$ & Fe II 58, 5835.4 \\
5874.1 & 7.6 & He I, 5875.6 \\
5891.4 & 0.9 & Na I, 5890.0 \\
5896.9 & 0.9 & Na I, 5895.6 \\
5952.4 & 0.6 & Fe III 115, 5953.7, unid -54.4 \\
5974.9 & 0.6 & Si II 4, 5979.0 \\
5987.9 & 3.2 & Fe II 46, 5991.4 \\
6040.6 & 0.4 & Fe II 46, 6044.5 \\
6080.8 & 1.2 & Fe II 46, 6084.1 \\
6125.1 & 0.7 & Fe II 46, 6129.7 \\
6145.8 & 3.3 & Fe II 74, 6147.7 \\
6234.8 & 2.5 & Fe II 74, 6238.4 \\
6243.9 & 4.2 & Fe II 74, 6247.6 \\
6314.7 & 1.2 & unid 6317.3 \\
6346.1 & 1.1 & Si II 2, 6347.1 \\
6367.8 & 1.6 & Si II 2, 6371.4, Fe II 40, -69.5 \\
6380.4 & 0.9 & Fe II -, 6383.7 \\
6414.0 & 1.6 & Fe II 74, 6416.9 \\
6429.4 & 3.6 & Fe II 40, 6432.7 \\
6453.9 & 4.0 & Fe II 74, 6456.4 \\
6488.6 & 1.2 & Fe II -, 6491.3 \\
6512.9 & 4.7 & Fe II 40, 6516.1 \\
6561.7 & 335 & H $\alpha$ \\
6676.7 & 2.3 & He I, 6678.1 \\
\hline & & \\
\hline & & \\
5 &
\end{tabular}

$I(\mathrm{H} \beta)=8.0 \times 10^{-10} \mathrm{erg} \mathrm{cm}^{-2} \mathrm{~s}^{-1}$. Extinction is corrected by $E(B-V)=0.57$. 
T. Iijima: Spectral evolution of the slowest classical nova V723 Cassiopeiae in the decline stage, Online Material p 8

Table 3. Intensities of emission lines relative to $\mathrm{H} \beta=100$ on January 28, 1997.

\begin{tabular}{|c|c|c|}
\hline $\begin{array}{l}\lambda_{\odot} \\
(\AA)\end{array}$ & $I(\lambda)$ & Identification \\
\hline $4615 \dagger$ & 1.6: & N II $5,4613.9$ \\
\hline $4629 \dagger$ & 6.0: & N II 5, 4630.5, Fe II 37, -29.3, N III 2, -34.2 \\
\hline 4639.1 & 5.9: & N III 2, 4640.6, -41.6, [Fe II] 4F, -39.7 \\
\hline 4656.1 & 6.4: & {$[\mathrm{Fe} \mathrm{III}] 3 \mathrm{~F}, 4658.1$} \\
\hline 4683.0 & 13.9: & He II, 4685.7 \\
\hline 4699.5 & 5.3: & [Fe III] 3F, 4701.5, He I, -13.2 \\
\hline 4727.8 & 2.1 & {$[\mathrm{Fe} \mathrm{II}]$ 4F, 4728.1} \\
\hline 4752.8 & 0.5 & {$[\mathrm{Fe}$ III] $3 \mathrm{~F}, 4754.7$} \\
\hline 4767.4 & 0.4 & {$[\mathrm{Fe}$ III] $3 \mathrm{~F}, 4769.4$} \\
\hline 4774.1 & 0.5 & [Fe II] 20F, 4774.7 \\
\hline 4813.4 & 1.2 & {$[\mathrm{Fe} \mathrm{II}]$ 20F, 4814.6, [Fe III] 3F, -13.9 } \\
\hline 4860.7 & 100 & $\mathrm{H} \beta$ \\
\hline 4882.4: & 2.6 & {$[\mathrm{Fe} \mathrm{III}] 2 \mathrm{~F}, 4881.0,[\mathrm{Fe} \mathrm{III}] \mathrm{1F},-83.9$} \\
\hline 4904.7 & 0.8 & {$[\mathrm{Fe}$ II $] 20 \mathrm{~F}, 4905.4$} \\
\hline 4922.1 & 4.6 & He I, 4921.9, Fe II 42, -23.9 \\
\hline 4951.4 & 0.6 & {$[\mathrm{Fe}$ II $] 20 \mathrm{~F}, 4950.7$} \\
\hline 5005.6 & 3.7 & [O III] 1F, 5006.8 \\
\hline 5015.8 & 6.7 & He I, 5015.7, Fe II 42, -18.4 \\
\hline 5039.9 & 2.2 & Si II 5, 5041.1 \\
\hline 5053.6 & 2.6 & Si II 5, 5056.0 \\
\hline 5108.5 & 0.7 & [Fe II] $18 \mathrm{~F}, 5108.0$ \\
\hline 5157.8 & 3.0 & {$[\mathrm{Fe} \mathrm{II}]$ 18F, 5158.0, [Fe II] 19F, -58.8} \\
\hline 5167.3 & 1.8 & Fe II $42,5169.0$ \\
\hline 5196.7 & 0.8 & Fe II 49, 5197.4 \\
\hline 5218.4 & 0.7 & [Fe II] 19F, 5220.1 \\
\hline 5232.5 & 0.9 & Fe II 49, 5234.6 \\
\hline 5262.3 & 2.3 & [Fe II] 19F, 5261.6 \\
\hline 5272.5 & 4.0 & [Fe II] 18F, 5273.4 \\
\hline 5315.4 & 2.6 & Fe II 49, 5316.6 \\
\hline 5332.2 & 1.0 & [Fe II] 19F, 5333.7 \\
\hline 5361.3 & 0.3 & Fe II 49, 5362.9, [Fe II] 17F, -62.1 \\
\hline 5374.7 & 0.6 & {$[\mathrm{Fe}$ II] $19 \mathrm{~F}, 5376.5$} \\
\hline 5411.2: & 2.4: & He II, 5411.5, [Fe II] 17F, -12.6 \\
\hline 5431.1 & 0.6 & {$[\mathrm{Fe}$ II $] 18 \mathrm{~F}, 5433.2$} \\
\hline 5476.3 & 0.2 & [Fe II] 34F, 5477.3, Fe II 49, -77.7 \\
\hline 5493.9 & 0.4 & N II 29, 5495.7, [Fe II] 17F, -95.8 \\
\hline 5526.9: & 0.9 & {$[\mathrm{Fe}$ II] $17 \mathrm{~F}, 5527.3,34 \mathrm{~F},-27.6,33 \mathrm{~F},-23.3$} \\
\hline 5534.2 & 0.4 & N II $63,5535.4$ \\
\hline $5672 \dagger$ & 2.2 & N II 3, 5666.6, -76.0, -79.6, -86.3 \\
\hline 5753.3 & 13.7 & {$[\mathrm{~N}$ II $] 3 \mathrm{~F}, 5754.8$} \\
\hline 5874.1 & 22.8 & He I, 5875.6 \\
\hline
\end{tabular}


T. Iijima: Spectral evolution of the slowest classical nova V723 Cassiopeiae in the decline stage, Online Material p 9

Table 3. continued.

\begin{tabular}{lrl}
\hline \hline $\begin{array}{l}\lambda_{\odot} \\
(\AA)\end{array}$ & $I(\lambda)$ & Identification \\
\hline 5956.2 & 0.6 & Si II 4, 5957.6, Fe II -, -56.5 \\
5977.6 & 0.5 & Si II 4, 5979.0 \\
6044.8 & 0.2 & Fe II 46, 6044.5 \\
6245.5 & 0.3 & Fe II 74, 6247.6 \\
6300.9 & 1.3 & [O I] 1F, 6300.2 \\
6314.1 & 1.4 & [S III] 3F, 6310.2, S II 26, -12.7 \\
6345.4 & 1.0 & Si II 2, 6347.1 \\
6368.1 & 0.6 & Si II 2, 6371.4, Fe II 40, -69.5 \\
6381.9 & 0.5 & Fe II -, 6383.7 \\
6455.1 & 0.5 & Fe II 74, 6456.4 \\
6484.2 & 0.4 & unid 6484.5 \\
6562.2 & 437 & H $\alpha$ \\
6675.8 & 8.7 & He I, 6678.1 \\
7062.7 & $12.8:$ & He I, 7065.2 \\
\hline
\end{tabular}

$\dagger$ : Diffuse emission hump.

Interstellar extinction is corrected by $E(B-V)=0.57$. 
T. Iijima: Spectral evolution of the slowest classical nova V723 Cassiopeiae in the decline stage, Online Material p 10

Table 6. Intensities of emission lines relative to $\mathrm{H} \beta=100$ in 1998 and 1999 measured with the Echelle spectrograph.

\begin{tabular}{|c|c|c|c|c|c|c|c|c|c|}
\hline \multirow[t]{3}{*}{$\lambda_{\odot}$} & \multirow{2}{*}{ 1998-1999 } & \multicolumn{8}{|c|}{$I(\lambda)$} \\
\hline & & Aug. 10 & Nov. 28 & Dec. 15 & Dec. 27 & Feb. 2 & Feb. 12 & Feb. 25 & Sep. 29 \\
\hline & JD $2450000+$ & 1035.6 & 1146.4 & 1163.4 & 1175.4 & 1212.3 & 1222.3 & 1235.3 & 1450.6 \\
\hline 4339.2 & $\mathrm{H} \gamma$ & 49.4 & 49.7 & & 51.2 & 47.9 & 46.9 & 45.8 & 46.1 \\
\hline 4361.3 & [O III] 63.0 & 18.2 & 17.9 & & 20.3 & 17.4 & 17.6 & 17.8 & 12.0 \\
\hline 4414.6 & [Fe II] 16.3 & 1.5 & & & 2.2 & 2.3 & & 2.4 & \\
\hline 4470.3 & He I 71.7 & 4.8 & 4.4 & & 3.5 & 4.0 & 4.4 & 3.4 & 4.1 \\
\hline 4513.1 & N III 14.9 & 1.6 & & & & & & & \\
\hline 4540.7 & He II 41.6 & 4.2 & 4.5 & & 4.4 & 5.2 & 4.9 & 5.0 & 7.2 \\
\hline 4605.3 & N II 07.2, [Fe III] 07.0 & 2.2 & & & 2.3 & 2.2 & & 2.2 & \\
\hline 4638.9 & N III 40.6, [Fe III] 39.7 & 3.6 & 3.5: & 2.9 & 3.2 & 2.9 & 2.6 & 2.4 & \\
\hline 4656.7 & {$[\mathrm{Fe}$ III $] 58.1$} & 3.2 & 3.8 & 3.1 & 3.6 & 3.3 & 3.6 & 3.4 & \\
\hline 4684.5 & He II 85.7 & 98.3 & 105.5 & 104.7 & 104.6 & 108.8 & 115.9 & 116.1 & 115.6 \\
\hline 4698.4 & [Fe VII] 99.0 & 3.1 & 4.0 & 3.5 & 3.6: & 4.4 & 4.4 & 5.6 & 4.8: \\
\hline 4712.8 & He I, [Ar IV]11.4, [Ne IV]14.0 & 5.0 & 5.2 & 5.4 & 6.0 & 5.4 & 5.9 & 6.3 & 3.4 \\
\hline 4723.9 & {$[\mathrm{Ne}$ IV] $24.2,25.7$} & 6.1 & 6.8 & 6.2 & 7.2 & 7.5 & 6.2 & 7.9 & 4.7 \\
\hline 4738.3 & [Ar IV] 40.3 & 0.8 & & 1.4 & & 0.9 & & & 2.5 \\
\hline 4754.5 & [Fe III $] 54.7$ & 0.6 & & & & & & & \\
\hline 4768.8 & [Fe III] 69.4 & 0.5 : & & & & & & & \\
\hline 4773.7 & {$[\mathrm{Fe}$ II] 74.4} & & & & & & & 1.0 & \\
\hline 4814.0 & [Fe II] 14.6 & 0.6 & & & & & & & \\
\hline 4860.2 & $\mathrm{H} \beta$ & 100 & 100 & 100 & 100 & 100 & 100 & 100 & 100 \\
\hline 4892.6 & [Fe VII] 93.9 & 4.2 & 5.0 & 4.6 & 5.7 & 6.1 & 6.4 & 6.2 & 8.6 \\
\hline 4903.6 & [Fe IV] 06.2, [Fe II] 05.4 & 2.5 & 2.8 & 2.8 & 2.7 & & 2.3 & 1.9 & \\
\hline 4921.3 & He I 21.9 & 1.4 & & 1.7 & 1.7 & & 1.3 & & \\
\hline 4940.4 & [Fe VII $] 42.3$ & 12.5 & 15.3 & 15.4 & 17.4 & 18.7 & 18.6 & 18.8 & 25.6 \\
\hline 4959.3 & [O III $] 58.9$ & 7.4: & 7.7 & 8.7 & 8.8 & 7.8 & 8.1 & 7.9 & 6.9 \\
\hline 4970.0 & {$[\mathrm{Fe} \mathrm{VI}] 68.8,74.0$} & 8.0 & 8.3 & 8.2 & 8.2 & 8.4 & 9.7 & 8.7 & 7.7 \\
\hline 4987.4 & [Fe VII] 89.4 & 2.7 & 3.4 & 3.1 & 3.5 & 4.2 & 4.9 & 4.3 & 7.4 \\
\hline 5005.8 & [O III] 06.9 & 20.0 & 21.7 & 24.1 & 23.7 & 19.4 & 21.4 & 20.7 & 16.4 \\
\hline 5014.8 & He I 15.7 & 1.6: & 1.4: & 1.9: & 2.4: & 3.6: & 1.7: & 1.7: & \\
\hline 5033.3 & [Fe II] 35.3 & 2.1: & & & 1.0: & 2.1: & & & \\
\hline 5040.4 & Si II 41.1, [Ni IV] 41.6 & 3.2 & 3.4 & 3.4 & 3.2 & 1.9: & 3.2 & 3.2 & 2.7 \\
\hline 5055.0 & Si II 56.0 & 1.7 & 1.6 & 1.1 & 1.2 & 1.2 & 1.2 & 1.3 & 1.6 \\
\hline 5111.6 & [Fe II] 11.6 & 0.4 & & & 0.8 & & & & \\
\hline 5144.6 & {$[\mathrm{Fe} \mathrm{VI}] 46.1$} & 6.7 & 6.8 & 6.5 & 7.4 & 7.7 & 7.6 & 6.9 & 7.2 \\
\hline 5157.8 & [Fe VII] 58.3 & 6.5 & 7.5 & 7.3 & 8.1 & 9.4 & 9.3 & 9.0 & 12.2 \\
\hline 5175.2 & {$[\mathrm{Fe} \mathrm{VI}] 77.0$} & 13.5 & 13.4 & 12.9 & 13.2 & 13.9 & 15.1 & 14.0 & 12.7 \\
\hline 5190.8 & [Ar III] 91.4 & 0.3 & & 0.3 & & 0.5 & & 0.4 & \\
\hline 5233.0 & [Fe IV] 33.8 & 2.6 & 2.1 & 2.2 & 1.8 & 1.6 & 2.1 & 2.0 & 1.6 \\
\hline 5261.6 & [Fe II] 61.6 & $0.8:$ & 1.7: & 1.8: & 1.3: & & & 1.0: & \\
\hline 5274.9 & [Fe VII $] 76.1$ & 9.6 & 8.7 & 8.7 & 9.6 & 9.9 & 9.9 & 10.5 & 12.2 \\
\hline 5308.2 & [Ca V] 08.9 & 12.8 & 13.4 & 13.5 & 13.5 & 14.3 & 14.2 & 14.3 & 12.5 \\
\hline 5333.6 & {$[\mathrm{Fe} \mathrm{VI}]$ 35.2, [Fe II] 33.7} & 1.4 & 1.3 & 1.9 & 1.4 & 1.5 & 2.0 & 1.5 & 2.1 \\
\hline 5410.2 & He II 11.5 & 7.7 & 10.5 & 9.3 & 9.9 & 10.1 & 9.9 & 10.0 & 10.9 \\
\hline 5424.1 & [Fe VI] 25.3 & 3.2 & 2.0 & 2.0 & 2.1 & 2.5 & 2.2 & 2.7 & 2.7 \\
\hline 5460.0 & [Ca VI] 60.6, N II 62.6 & 1.1 & 1.2 & 1.1 & & & & & 1.1: \\
\hline 5485.1 & {$[\mathrm{Fe} \mathrm{VI}] 85.7$} & 0.8 & & 0.7 & 0.9 & & 1.0 & 0.9 & \\
\hline 5525.1 & {$[\mathrm{Fe}$ II] $23.3,27.3$} & 0.6 & & & & & & & \\
\hline
\end{tabular}


T. Iijima: Spectral evolution of the slowest classical nova V723 Cassiopeiae in the decline stage, Online Material p 11

Table 6. continued.

\begin{tabular}{|c|c|c|c|c|c|c|c|c|c|}
\hline \multirow[t]{3}{*}{$\lambda_{\odot}$} & \multirow[b]{3}{*}{ JD $2450000+$} & \multicolumn{8}{|c|}{$I(\lambda)$} \\
\hline & & Aug. 10 & Nov. 28 & Dec. 15 & Dec. 27 & Feb. 2 & Feb. 12 & Feb. 25 & Sep. 29 \\
\hline & & 1035.6 & 1146.4 & 1163.4 & 1175.4 & 1212.3 & 1222.3 & 1235.3 & 1450.6 \\
\hline 5582.1 & [Fe II] $82.0,[\mathrm{Ca}$ VI] 87.2 & 0.5 & 0.7 & & & 0.7 & & 0.7 & \\
\hline 5617.2 & [Ca VII] 18.8 & 6.2 & 7.8 & 9.4 & 10.0 & 10.4 & 11.1 & 11.6 & 15.2 \\
\hline 5629.9 & {$[\mathrm{Fe} \mathrm{VI}] 31.6,[\mathrm{Ca} \mathrm{VI}] 31.0$} & 1.6: & 1.9 & 1.7 & 1.6: & 1.5 & 1.8: & 2.5 & 2.6: \\
\hline 5675.7 & {$[\mathrm{Fe} \mathrm{VI}] 78.0$} & 2.0 & 2.3 & 2.1 & 1.9 & 2.7 & 2.5 & 2.5 & 2.9 \\
\hline 5719.9 & [Fe VII $] 20.9$ & 34.9 & 38.3 & 37.0 & 39.2 & 42.7 & 49.1 & 46.0 & 54.2 \\
\hline 5753.1 & [N II] 54.8 & 10.4 & 10.6 & 12.7 & 11.0 & 11.0 & 11.3 & 11.1 & 10.3 \\
\hline 5800.0 & C IV 01.5 & 0.9 & 0.9 & 1.0 & 1.3 & 1.1: & 1.7 & 0.6 : & 1.7 \\
\hline 5874.2 & He I 75.6 & 15.1 & 11.1 & 11.4 & 10.2 & 10.1 & 11.0 & 10.5 & 7.9 \\
\hline 6036.1 & He II 36.7 & & & 0.2 & & 0.5 & & & \\
\hline 6085.4 & {$[\mathrm{Fe} \mathrm{VII}]$ 85.5, [Ca V] 85.9} & 54.2 & 60.3 & 58.4 & 59.9 & 66.7 & 80.5 & 73.3 & 84.8 \\
\hline 6118.2 & He II 18.2 & 0.4 & & & & & & & \\
\hline 6229.7 & He II 33.8 & 0.6 & 0.5 & 0.6 & 0.8 & 0.9 & 0.8 & 1.0 & \\
\hline 6310.1 & [S III] 10.2, S II 12.7 & 4.3 & 4.6 & 4.6 & 2.7 & 4.1 & 4.3 & 4.0 & 4.5 \\
\hline 6345.2 & Si II 47.1 & 0.6 & 0.7 & 0.6 & 0.6 & 0.4 & 0.5 & 0.4 & \\
\hline 6369.5 & Si II 71.4 & 1.1 & 0.8 & 1.2 & 1.3 & 0.7 & 0.9 & 0.9 & 0.8 \\
\hline 6404.6 & He II 06.3 & 0.5 & 0.5 & 0.6 & 0.6 & 0.5 & 0.9 & 0.5 & 0.6 \\
\hline 6433.4 & [Ar V] 34.9 & 2.6 & 2.7 & 2.9 & 2.9 & 2.9 & 3.4 & 3.3 & 2.9 \\
\hline 6525.4 & [N II] 27.4, He II 27.3 & 0.5 & & 0.4 & & & 0.6 & 0.6 & 0.8 \\
\hline 6561.3 & $\mathrm{H} \alpha$ & 269 & & 269 & 271 & 269 & 279 & 283 & 257 \\
\hline 6582.3 & [N II] 83.6 & 1.2 & 1.4 & 1.7 & 2.5: & 1.5 & 2.3: & 1.6 & 1.9 \\
\hline 6677.2 & He I 78.2 & 4.3 & 3.7 & 3.2 & 3.7 & 3.4 & 3.8 & 3.3 & 2.6 \\
\hline 6791.2 & [K IV] 94.8 & $0.5:$ & & 0.5 & & 0.4 & 0.6 & 0.3 & \\
\hline 6819.5 & [Fe VI] 20.6 & $0.5:$ & & & & 0.3 & & 0.3 & \\
\hline 7002.7 & [Ar V] 06.3 & & & 6.7: & & & & & \\
\hline 7063.3 & He I 65.2 & & & 7.6 & & & & & \\
\hline
\end{tabular}

Interstellar extinction is corrected by $E(B-V)=0.57$. 
T. Iijima: Spectral evolution of the slowest classical nova V723 Cassiopeiae in the decline stage, Online Material p 12

Table 7. Intensities of emission lines relative to $\mathrm{H} \beta=100$ in 1998 and 1999 measured with the $\mathrm{B} \& \mathrm{C}$ spectrograph.

\begin{tabular}{|c|c|c|c|c|c|c|c|c|}
\hline \multirow[t]{3}{*}{$\lambda_{\odot}$} & \multirow{3}{*}{$\begin{array}{l}1998-1999 \\
\text { JD } 2450000\end{array}$} & \multicolumn{7}{|c|}{$I(\lambda)$} \\
\hline & & Aug. 22 & Oct. 11 & Oct. 16 & Nov. 17 & Jan. 5,6 & Jan. 25 & Nov. 28 \\
\hline & & 1047.6 & 1097.6 & 1102.7 & 1135.4 & $1184-5$ & 1204.3 & $1511-2$ \\
\hline 3424.5 & {$[\mathrm{Ne} \mathrm{V}] 25.8$} & & & 346 & & & & \\
\hline 3488.9 & {$[\mathrm{Fe}$ II $] 90.0$} & & & 19.4 & & & & \\
\hline 3585.7 & [Fe VII $] 87.2$ & & & 54.1 & & & & \\
\hline 3661.7 & {$[\mathrm{Fe} \mathrm{VI}] 64.1$} & & & 9.1 & & & & \\
\hline 3726.7 & [S III]21.1, [O II]26.2, 28.9, [Ni V]26.7 & & & 12.2 & & & & 28.2 \\
\hline 3758.6 & [Fe VII] 59.9, [Ni V] 57.2 & & & 81.6 & & & & 116.4 \\
\hline 3797.7 & H 10, [S III] 96.7, [Fe V] 98.2 & & & 10.1 & & & & 11.4 \\
\hline 3812.4 & He II 13.5 & & & 6.5 & & & & 6.1 \\
\hline 3835.8 & $\mathrm{H} 9,[\mathrm{Fe} \mathrm{V}] 38.1$ & & & 17.9 & & & & 20.7 \\
\hline 3867.6 & {$[\mathrm{Ne}$ III] 68.7} & & & 91.3 & & & & 70.4 \\
\hline 3889.6 & $\mathrm{H} \mathrm{8,} \mathrm{[Fe} \mathrm{VI]} \mathrm{90.9,} \mathrm{[Fe} \mathrm{V]} 91.8$ & & & 49.0 & & & & 44.8 \\
\hline 3967.4 & $\mathrm{H} \epsilon,[\mathrm{Ne} \mathrm{III}] 67.5$ & 58.1 & & 47.6 & 51.9 & & & 43.1 \\
\hline 3993.4 & {$[\mathrm{Fe} \mathrm{VI}] 95.8,[\mathrm{Ca} \mathrm{V}] 96.3$} & & & & & & & 4.3 \\
\hline 4001.1 & {$[\mathrm{Fe} \mathrm{V}] 03.2$} & 3.9 & & & & & & 4.6 \\
\hline 4024.8 & He II 25.6, He I 26.2 & 4.8 & & 4.2 & 4.9 & 5.4 & & 5.3 \\
\hline 4070.6 & {$[\mathrm{Fe} \mathrm{V}] 71.5$} & 11.5 & 11.5 & 10.0 & 11.7 & 10.2 & 11.2 & 9.7 \\
\hline 4100.7 & $\mathrm{H} \delta, \mathrm{N}$ III 97.3, 03.4 & 38.2 & 42.2 & 34.1 & 38.0 & 36.5 & 38.1 & 36.6 \\
\hline 4141.4 & He I 43.8, [Fe IV]44.4, [Fe II]46.7 & 4.1 & 5.1 & 4.4 & 3.5 & 3.2 & 4.1 & 2.6 \\
\hline 4179.5 & {$[\mathrm{Fe} \mathrm{V}]$ 81.3, [Fe II] 77.2, 79.0} & 2.8 & 3.7 & 3.5 & 3.1 & 3.4 & 2.7 & 2.4 \\
\hline 4199.5 & He II 99.8, [Ni II] 01.7, N III 0.0 & 6.3 & 7.9 & 6.8 & 7.3 & 8.8 & 8.1 & 9.2 \\
\hline 4244.6 & {$[\mathrm{Fe} \mathrm{II}] 44.0,44.8$} & 4.9 & 4.6 & 5.6 & 5.8 & 7.4 & 6.2 & 6.9 \\
\hline 4275.9 & {$[\mathrm{Fe}$ II $] 76.8$} & 1.5 & 1.4 & & 1.6 & & & \\
\hline 4286.4 & [Fe II] 87.4 & 1.5 & 1.5 & 2.1 & 2.0 & & & 1.8 \\
\hline 4339.2 & $\mathrm{H} \gamma$ & 56.6 & 57.8 & 52.1 & 57.4 & 55.9 & 57.7 & 56.5 \\
\hline 4361.3 & [O III] 63.0 & 20.8 & 20.1 & 17.9 & 20.3 & 19.0 & 18.8 & 15.1 \\
\hline 4414.1 & [Fe II] $13.8,14.5,16.3$ & 1.9 & 2.3 & 1.4 & 1.4 & 1.8 & 2.1 & 3.0 \\
\hline 4470.4 & He I 71.7 & 5.2 & 5.9 & 5.1 & 4.1 & 4.7 & 4.6 & 3.4 \\
\hline 4540.1 & He II 41.6 & 4.4 & 5.6 & 5.5 & 4.6 & 5.3 & 5.9 & 6.3 \\
\hline 4604.5 & N II 07.3, [Fe III] 07.0 & 2.0 & 3.1 & 2.8 & 1.8 & 2.5 & 2.3 & 2.8 \\
\hline 4638.3 & N III 39.7, 40.6, 41.9, [Fe II] 39.7 & 6.6 & 7.3 & 6.8 & 3.6 & 4.3 & 4.9 & 2.5: \\
\hline 4655.8 & [Fe III] 58.1, C IV 58.6 & 4.8 & 5.1 & 4.2 & 3.8 & 4.0 & 3.8 & 5.2: \\
\hline 4684.7 & He II 85.7 & 103.3 & 114.8 & 109.9 & 111.7 & 117.3 & 119.6 & 128.0 \\
\hline 4712.1 & He I 13.4, [Ne IV] 14.0, [Ar IV] 11.4 & 4.2: & 5.9: & 4.9: & 4.8: & 4.9: & 4.4: & 3.9: \\
\hline 4723.2 & {$[\mathrm{Ne} I V] 24.2,25.7$} & 6.4: & 4.9: & 6.6: & 5.8: & 5.5: & 6.3: & 6.0: \\
\hline 4860.3 & $\mathrm{H} \beta$ & 100 & 100 & 100 & 100 & 100 & 100 & 100 \\
\hline 4893.3 & [Fe VII] 93.9 & $6.5 \dagger$ & $7.4 \dagger$ & 4.2: & 4.3: & $7.0 \dagger$ & $7.9 \dagger$ & $11.9 \dagger$ \\
\hline 4904.1 & {$[\mathrm{Fe}$ IV] $06.2,[\mathrm{Fe} \mathrm{II}] 05.4$} & & & 2.8: & 2.5: & & & \\
\hline 4921.9 & He I 21.9, [Fe III] 24.5 & & & 1.3 & & & 0.8: & 1.1 \\
\hline 4940.4 & {$[\mathrm{Fe} \mathrm{VII}] 42.3$} & 10.9 & 14.7 & 14.6 & 14.8 & 17.6 & 18.5 & 27.5 \\
\hline 4965.5 & {$[\mathrm{Fe} \mathrm{VI}] 68.8,[\mathrm{O} \mathrm{III}] 58.9$} & 13.6 & 16.8 & 16.5 & 14.0 & 16.4 & 17.5 & 20.0 \\
\hline 4987.3 & [Fe VII] 89.4 & 2.1 & 2.9 & 2.4 & 3.1 & 2.9 & 4.3 & 8.3 \\
\hline 5005.9 & [O III $] 06.8$ & 23.8 & 21.0 & 20.8 & 23.6 & 21.3 & 23.5 & 25.2 \\
\hline 5039.6 & Si II 41.1, [Ni IV] 41.4 & 4.7 & 4.0 & 3.8 & 3.3 & 3.4 & 2.7 & 2.0 \\
\hline 5057.9 & Si II 56.0, [Fe III] 60.3 & 1.6 & 1.5 & 1.2 & 1.1 & 1.1 & 1.1 & 0.8 \\
\hline 5144.9 & {$[\mathrm{Fe} \mathrm{VI}] 46.8$} & & & 7.4 & & 6.2 & 6.7 & 7.7 \\
\hline 5157.7 & [Fe VII $] 58.3$ & & & 7.3 & & 7.2 & 8.0 & 14.2 \\
\hline 5175.3 & {$[\mathrm{Fe} \mathrm{VI}] 77.0$} & & & 14.6 & & 13.1 & 14.4 & 15.9 \\
\hline
\end{tabular}


T. Iijima: Spectral evolution of the slowest classical nova V723 Cassiopeiae in the decline stage, Online Material p 13

Table 7. continued.

\begin{tabular}{|c|c|c|c|c|c|c|c|c|}
\hline \multirow[t]{3}{*}{$\lambda_{\odot}$} & ID & \multicolumn{7}{|c|}{$I(\lambda)$} \\
\hline & 1998-1999 & Aug. 21 & Oct. 11 & Oct. 16 & Nov. 17 & Jan. 5,6 & Jan. 25 & Nov. 28 \\
\hline & JD $2450000+$ & 1046.6 & 1097.6 & 1102.7 & 1135.4 & $1184-5$ & 1204.3 & $1511-2$ \\
\hline 5232.3 & {$[\mathrm{Fe}$ IV] 33.9} & & & 1.8 & & 1.6 & & 1.8 \\
\hline 5274.2 & {$[\mathrm{Fe} \mathrm{VII}] 76.1$} & & & 8.4 & & 10.0 & & 15.5 \\
\hline 5307.9 & {$[\mathrm{Ca} \mathrm{V}] 08.9$} & & & 13.4 & & 13.8 & & 15.4 \\
\hline 5332.5 & [Fe II] 33.7, [Fe VI] 36.4 & & & 1.4 & & 1.5 & & 1.9 \\
\hline 5410.4 & He II 11.5 & & & 9.7 & & 10.2 & & 14.3 \\
\hline 5424.5 & {$[\mathrm{Fe} \mathrm{VI}] 25.3,28.6$} & & & 1.8 & & 1.4 & & 2.4 \\
\hline 5458.7 & [Ca VI] 60.6, N II 62.6 & & & 1.1 & & 1.0 & & 0.7 \\
\hline 5485.8 & {$[\mathrm{Fe} \mathrm{VI}] 85.7$} & & & 0.6 & & & & 0.9 \\
\hline 5585.8 & [Ca VI] 87.2 & & & 0.7 & & 0.8 & & \\
\hline 5617.6 & [Ca VII] 15.8 & & & 8.3 & & 10.6 & & 17.7 \\
\hline 5631.2 & {$[\mathrm{Fe} \mathrm{VI}] 31.6,[\mathrm{Ca} \mathrm{VI}] 31.0$} & & & 1.4 & & 0.8 & & 1.2 \\
\hline 5675.4 & {$[\mathrm{Fe} \mathrm{VI}] 78.0, \mathrm{~N}$ II 79.6} & & & 1.9 & & 2.0 & & 3.4 \\
\hline 5719.7 & [Fe VII $] 20.9$ & 32.0 & & 37.5 & & 38.2 & & 60.5 \\
\hline 5753.1 & [N II] 54.8 & 11.6 & & 10.4 & & 9.1 & & 12.8 \\
\hline 5800.5 & C IV 01.5 & 0.9 & & 1.1 & & 0.9 & & \\
\hline 5807.7 & [Fe II] 09.4 & & & & & & & 1.4 \\
\hline 5874.1 & He I 75.6 & 13.7 & & 13.5 & & 10.0 & & 7.7 \\
\hline 6085.4 & {$[\mathrm{Fe}$ VII $]$ 85.5, [Ca V] 85.9} & 50.3 & & 59.5 & & 62.0 & & 92.8 \\
\hline 6229.2 & He II 33.8, [K VI] 29.2 & 0.6 & & 0.8 & & 0.8 & & 1.1 \\
\hline 6310.1 & [S III] 10.2, S II 12.7 & 4.0 & & 3.9 & & 3.8 & & 4.4 \\
\hline 6345.0 & Si II 47.1, [K V] 49.5 & 0.5 & & 0.6 & & 0.4 & & 0.7 \\
\hline 6369.2 & Si II 71.4 & 1.1 & & 1.1 & & 1.0 & & \\
\hline 6374.3 & {$[\mathrm{Fe} \mathrm{X}] 74.5$} & & & & & & & 1.9 \\
\hline 6403.5 & He II 06.3 & 0.5 & & 0.5 & & 0.5 & & 1.0 \\
\hline 6433.3 & [Ar V] 34.9 & 2.0 & & 2.6 & & 2.8 & & 3.2 \\
\hline 6561.1 & $\mathrm{H} \alpha$ & 243 & & & & 235 & & 277 \\
\hline 6583.4 & [N II] 83.6 & 1.2: & & & & 1.4: & & 3.3: \\
\hline 6678.4 & He I 78.1 & 3.5 & & & & 2.8 & & 2.9 \\
\hline 6792.0 & [K IV] 94.8 & 0.6 & & & & 0.5 & & \\
\hline 6888.9 & He II 90.9 & & & & & 0.7 & & 0.9 \\
\hline 7002.9 & [Ar V] 06.3 & & & & & 5.6 & & 7.0 \\
\hline 7063.0 & He I 65.2 & & & & & 5.4 & & 4.9 \\
\hline 7142.3 & [Ar III] 35.8, [Ti III] 52.8 & & & & & & & 4.3 \\
\hline \multirow[t]{2}{*}{7171.9} & [Fe II] 72.0, [Ar IV] 69.0 & & & & & & & 3.1 \\
\hline & $\mathrm{I}(\mathrm{H} \beta)$ in $10^{-12} \mathrm{erg} \mathrm{cm}^{-2} \mathrm{~s}^{-1}$ & $37.7 \ddagger$ & 32.2 & 31.6 & 30.1 & 25.3 & 25.4 & 9.7 \\
\hline
\end{tabular}

$\ddagger: I(\mathrm{H} \beta)$ on August 22, 1998, $\uparrow:$ blended by [Fe IV] 06.2 and $[\mathrm{Fe} I \mathrm{II}]$ 05.4. Extinction is corrected by $E(B-V)=0.57$. 
T. Iijima: Spectral evolution of the slowest classical nova V723 Cassiopeiae in the decline stage, Online Material p 14

Table 8. Intensities of emission lines relative to $\mathrm{H} \beta=100$ or $\mathrm{H} \alpha=270$ in 2000 and 2001 measured with the $\mathrm{B} \& \mathrm{C}$ spectrograph.

\begin{tabular}{|c|c|c|c|c|c|c|c|c|}
\hline \multirow[t]{3}{*}{$\lambda_{\odot}$} & \multirow{3}{*}{$\begin{array}{l}2000-2001 \\
\text { JD } 2450000+\end{array}$} & \multicolumn{7}{|c|}{$I(\lambda)$} \\
\hline & & Feb. 5 & Feb. 11 & Feb. 16 & Feb. 18 & Sep. 27 & Oct. 14,15 & Dec. 11 \\
\hline & & 1580.4 & 1952.3 & 1957.3 & 1959.3 & 2179.6 & 2196-7 & 2255.5 \\
\hline 4070.6 & {$[\mathrm{Fe} \mathrm{V}] 71.5$} & & & 15.7 & & & 23.7 & \\
\hline 4100.7 & $\mathrm{H} \delta, \mathrm{N}$ III 97.3, 03.4 & & & 44.3 & & 42.1 & 53.3 & \\
\hline 4141.4 & He I 43.8, [Fe IV]44.4, [Fe II]46.7 & & & 7.2 & & & 13.2 & \\
\hline 4199.5 & He II 99.8, [Ni II] 01.7, N III 0.0 & & & 14.4: & & & 13.4 & \\
\hline 4244.6 & {$[\mathrm{Fe} \mathrm{II}] 44.0,44.8$} & & & 6.3: & & & & \\
\hline 4339.2 & $\mathrm{H} \gamma$ & & & 66.3 & & 67.8 & 65.0 & \\
\hline 4361.3 & [O III] 63.0 & & & 14.2 & & 8.8: & 8.0: & \\
\hline 4414.1 & {$[\mathrm{Fe}$ II $] 13.8,14.5,16.3$} & & & & & & & \\
\hline 4470.4 & He I 71.7 & & & 3.5: & & & & \\
\hline 4540.1 & He II 41.6 & & & 5.6 & & & 9.1 & \\
\hline 4604.5 & N II 07.2, [Fe III] 07.0 & & & & & & 4.1 & \\
\hline 4638.3 & N III 39.7, 40.6, 41.9, [Fe II] 39.7 & & & 3.9 & & & 4.4 & \\
\hline 4655.8 & [Fe III] 58.1, C IV 58.6 & & & 4.6 & & & 6.2 & \\
\hline 4684.7 & He II 85.7 & & & 152.9 & & 138.2 & 143.4 & \\
\hline 4712.1 & He I 13.4, [Ne IV] 14.0, [Ar IV] 11.4 & & & 5.5: & & & & \\
\hline 4723.2 & {$[\mathrm{Ne} I V] 24.2,25.7$} & & & 7.3: & & & & \\
\hline 4860.3 & $\mathrm{H} \beta$ & & & 100 & & 100 & 100 & \\
\hline 4893.0 & [Fe VII] 93.9 & & & $9.9 \dagger$ & & $9.8 \dagger$ & 8.1 & \\
\hline 4904.1 & {$[\mathrm{Fe} \mathrm{IV]} \mathrm{06.2,} \mathrm{[Fe} \mathrm{II]} 05.4$} & & & & & & 3.3 & \\
\hline 4921.9 & He I 21.9, [Fe III $] 24.5$ & & & 1.7 & & 3.9 & 3.1 & \\
\hline 4940.4 & [Fe VII $] 42.3$ & & & 29.3 & & 33.2 & 26.2 & \\
\hline 4965.5 & [Fe VI] 68.8, [O III] 58.9 & & & 16.4 & & 11.7 & 5.7 & \\
\hline 4987.3 & [Fe VII] 89.4 & & & 10.3 & & 13.0 & 10.6 & \\
\hline 5005.9 & [O III] 06.8 & & & 20.3 & & 24.2 & 16.0 & \\
\hline 5017.6 & He I 15.7 & & & & & & 4.2 & \\
\hline 5039.6 & Si II 41.1, [Ni IV] 41.4 & & & 2.2 & & & & \\
\hline 5144.9 & {$[\mathrm{Fe} \mathrm{VI}] 46.8$} & & & 5.8 & & 4.5: & 5.1: & \\
\hline 5157.7 & [Fe VII $] 58.3$ & & & 15.4 & & 13.9: & 14.2 & \\
\hline 5175.3 & {$[\mathrm{Fe} \mathrm{VI}] 77.0$} & & & 12.3 & & & & \\
\hline 5874.1 & He I 75.6 & 7.1 & 5.7 & & & & 6.6 & \\
\hline 6085.4 & [Fe VII] 85.5, [Ca V] 85.9 & 87.6 & 90.7 & & 92.7 & & 85.6 & 88.3 \\
\hline 6229.2 & He II 33.8, [K VI] 29.2 & 1.1 & 1.2 & & 1.1 & & & 2.8 \\
\hline 6310.1 & [S III] 10.2, S II 12.7 & 3.7 & 3.9 & & 3.7 & & 4.9 & 5.8 \\
\hline 6345.0 & Si II 47.1, [K V] 49.5 & 0.5 & & & & & & \\
\hline 6374.3 & {$[\mathrm{Fe} \mathrm{X}] 74.5$} & 2.1 & 3.4 & & 2.7 & & 10.9 & 12.1 \\
\hline 6403.5 & He II 06.3 & 0.7 & 1.0 & & 1.7 & & & \\
\hline 6433.3 & [Ar V] 34.9 & 3.0 & 3.3 & & 2.8 & & 2.8 & 3.5 \\
\hline 6561.1 & $\mathrm{H} \alpha$ & 270 & 270 & & 270 & & 260 & 270 \\
\hline 6583.4 & [N II] 83.6 & 2.4: & 2.7 & & 3.3 & & 5.3 & 4.4 \\
\hline 6678.4 & He I 78.1 & 2.6 & 2.4 & & 1.8 & & 2.0 & 3.1: \\
\hline 6888.9 & He II 90.9 & 0.6 & & & & & & \\
\hline 7002.9 & [Ar V] 06.3 & & & & 5.9 & & & 4.2 \\
\hline 7063.0 & He I 65.2 & & & & 2.8: & & & 2.3 \\
\hline \multirow[t]{2}{*}{7142.3} & [Ar III] 35.8, [Ti III] 52.8 & & & & & & & 3.6 \\
\hline & $\mathrm{I}(\mathrm{H} \beta)$ in $10^{-12} \mathrm{erg} \mathrm{cm}^{-2} \mathrm{~s}^{-1}$ & & & 3.6 & & & $2.4 \ddagger$ & \\
\hline
\end{tabular}

$\ddagger: I(\mathrm{H} \beta)$ on October 14, 2001, $\uparrow:$ blended by [Fe IV] 06.2 and $[\mathrm{Fe} \mathrm{II}]$ 05.4. Extinction is corrected by $E(B-V)=0.57$. 